\title{
PDMM: A novel Primal-Dual Majorization-Minimization algorithm for Poisson Phase-Retrieval problem
}

\author{
Ghania Fatima, Zongyu Li, Aakash Arora, and Prabhu Babu
}

\begin{abstract}
In this paper, we introduce a novel iterative algorithm for the problem of phase-retrieval where the measurements consist of only the magnitude of linear function of the unknown signal, and the noise in the measurements follow Poisson distribution. The proposed algorithm is based on the principle of majorization-minimization (MM); however, the application of MM here is very novel and distinct from the way MM has been usually used to solve optimization problems in the literature. More precisely, we reformulate the original minimization problem into a saddle point problem by invoking Fenchel dual representation of the $\log ($.$) term in the Poisson likelihood$ function. We then propose tighter surrogate functions over both primal and dual variables resulting in a double-loop MM algorithm, which we have named as Primal-Dual MajorizationMinimization (PDMM) algorithm. The iterative steps of the resulting algorithm are simple to implement and involve only computing matrix vector products. We also extend our algorithm to handle various $\ell_{1}$ regularized Poisson phase-retrieval problems (which exploit sparsity). The proposed algorithm is compared with previously proposed algorithms such as wirtinger flow (WF), MM (conventional), and alternating direction methods of multipliers (ADMM) for the Poisson data model. The simulation results under different experimental settings show that PDMM is faster than the competing methods, and its performance in recovering the original signal is at par with the state-of-the-art algorithms.
\end{abstract}

Index Terms-Phase-retrieval, Poisson data model, Majorization-Minimization (MM), Saddle-point problem, Fenchel dual representation.

\section{INTRODUCTION AND LITERATURE}

Many physical measurement systems measure only the magnitude (or magnitude square) of the signal and not its phase. For example, optical devices (e.g., CCD cameras, photosensitive films) cannot directly measure the phase of the light wave because of the high rate of electromagnetic field oscillations $\left(\sim 10^{15} \mathrm{~Hz}\right)$ and measure only the photon flux, which is the magnitude square of the electromagnetic field [1]. At a large enough distance from the imaging plane, the electromagnetic field structure is given by the product of the Fourier transform of the image and a known phase factor. It is

Ghania Fatima and Prabhu Babu are with CARE, IIT Delhi, New Delhi, India (e-mail: Ghania.Fatima@care.iitd.ac.in, prabhubabu@care.iitd.ac.in).

Zongyu $\mathrm{Li}$ is with Department of Electrical Engineering and Computer Science, University of Michigan, Ann Arbor, MI $48109-2122$ (e-mail: zonyul@umich.edu).

Aakash Arora is with SnT, University of Luxembourg, Luxembourg (e-mail: aakash.arora@uni.lu).

Zongyu Li is funded by NSF grant IIS 1838179, and NIH grants R01 EB022075 and R01 CA240706. known that in the Fourier representation of signals, the magnitude and phase play different roles [2], and in many cases, the important features of the signal are preserved only in the phase. Since important phase information is lost, the recorded signal does not resemble the original signal, and recovering the phase from the Fourier magnitude is a challenging task. Therefore, it is important to come up with sturdy algorithms that successfully retrieve the original signal with only the magnitude information at hand. The problem of recovering the original signal from the magnitude (or magnitude square) of its linear measurements is called phase-retrieval problem. Various fields in which the problem of phase-retrieval arises are optical imaging [3], astronomical imaging [4], speech and audio processing [5], [6], [7], [8], crystallography [9], [10], [11], computational biology [12], and electron microscopy [13], [14], [15].

Mathematically, phase retrieval is to obtain a $K$-dimensional complex valued signal $\mathrm{x}$ from $N$ measurements denoted by $\mathbf{y} \in \mathbb{R}_{+}^{N}$, which are nothing but magnitude squares of some linear function of $\mathrm{x}$ and are modelled as:

$$
y_{i}=\left|\mathbf{a}_{i}^{\mathrm{H}} \mathbf{x}\right|^{2}+b_{i}, i=1, \ldots, N
$$

The measurement vectors $\left\{\mathbf{a}_{i}^{\mathrm{H}}\right\}_{i=1}^{N} \in \mathbb{C}^{K}$ that corresponds to the rows of the measurement matrix $\mathbf{A} \in \mathbb{C}^{N \times K}$ (assumed to have full column rank), and the mean background signal $b_{i} \in$ $\mathbb{R}_{+}$for the $i^{\text {th }}$ measurement are usually known beforehand. In the cases where $y_{i}^{\prime} \mathrm{s}$ correspond to magnitude square of the Fourier transform of $\mathbf{x}, \mathbf{a}_{i}^{\mathrm{H}}$ would be the rows of Discrete Fourier Transform (DFT) matrix. Often the measurements are corrupted with noise and thus (1) becomes

$$
y_{i}=\left|\mathbf{a}_{i}^{\mathrm{H}} \mathbf{x}\right|^{2}+b_{i}+n_{i}, i=1, \ldots, N,
$$

where $n_{i}$ denotes the measurement noise.

Since important phase information is lost, the number of measurements $(N)$ should be in general larger than the dimension of the signal $(K)$ for the stable recovery of the signal. The authors of [16] proved that $N$ should at least be of the order of $K \log K$ in order to successfully recover the original signal. Furthermore, the authors of [17] proved that $N=4 K-4$ is necessary and sufficient to uniquely recover the original signal.

Most of the previous works done on phase-retrieval assumes the data model to be Gaussian where the entries of $\mathbf{y}$ are statistically independent and follow Gaussian distribution, i.e.,

$$
y_{i} \sim \mathcal{N}\left(\left|\mathbf{a}_{i}^{\mathrm{H}} \mathbf{x}\right|^{2}+b_{i}, \sigma^{2}\right)
$$


where $\sigma^{2}$ denotes the variance of the noise in the measurements. The maximum likelihood (ML) estimate of $\mathbf{x}$ in this case would be obtained by solving the following non-convex problem:

$$
\left.\left.\min _{\mathbf{x}} \sum_{i=1}^{N}\left|y_{i}-b_{i}-\right| \mathbf{a}_{i}^{\mathrm{H}} \mathbf{x}\right|^{2}\right|^{2}
$$

A number of algorithms have been previously proposed to solve for a minimizer of (4). One of the recent approaches involves the reformulation of the phase-retrieval problem by a technique called "matrix-lifting" [16], [18], [19], wherein a rank-1 matrix $\mathbf{X}:=\mathbf{x x}^{\mathrm{H}}$ is introduced and the problem is transformed into a higher dimensional space making the Fourier magnitude square measurements linear in $\mathbf{X}$. Although the objective of the transformed problem is convex, the resulting problem is non-convex due to the presence of rank-1 constraint, which is then relaxed and the resultant problem is solved using semi-definite programming (SDP). The SDP based algorithm yields robust solutions but is computationally demanding, reducing their applications to only low dimension problems. In the classical WF [20] algorithm for phaseretrieval, the step size is updated in an ad-hoc manner to descend the cost function in (4). The step size is initially kept small and then increased with each iteration, thereby requiring a hyper parameter for its control. PRIME [21] uses the MM technique wherein a sequence of some surrogate problems are solved instead of the original non-convex problem yielding a simple iterative algorithm.

The authors of [22], [23], [24] proposed algorithms for phase-retrieval problem based on the magnitude model instead of the intensity (or squared magnitude) model in (1). In [22], the authors have proposed an algorithm known as GerchbergSaxton (GS) algorithm, wherein a new auxiliary variable is introduced to reformulate the problem and the equivalent problem is then solved by alternating minimization. In [23], a phase-retrieval algorithm using ADMM was proposed wherein auxiliary magnitude and phase variables are introduced to eliminate the absolute value operator. More recently, the authors of [24] proposed an iterative soft-thresholding with exact line search algorithm (STELA) for sparse phase-retrieval which is based on the successive convex approximation (SCA) framework. The authors of [25] combined the ideas of SDP relaxation and GS algorithm to come up with a novel method that lifts phase-vector instead of the original signal $\mathbf{x}$ and deduced convex relaxation for the non-convex problem which was then solved using block coordinate descent method.

Although the assumption of Gaussian noise in the measurements is common, in low photon count applications such as coherent diffractive imaging [26], ptychography [27], [28] and holographic phase retrieval [29], the light source is weak and consequently, the detection of SNR is limited by the quantized nature of light, wherein the shot noise cannot be avoided [30]. Therefore in such cases, assuming Poisson distribution to model the noise is more appropriate, i.e.,

$$
y_{i} \sim \text { Poisson }\left(\left|\mathbf{a}_{i}^{\mathrm{H}} \mathbf{x}\right|^{2}+b_{i}\right)
$$

The ML estimate of $\mathbf{x}$ for the Poisson data model can be obtained by solving the following non-convex problem:

$$
\min _{\mathbf{x}} \sum_{i=1}^{N}\left[\left|\mathbf{a}_{i}^{\mathrm{H}} \mathbf{x}\right|^{2}+b_{i}-y_{i} \log \left(\left|\mathbf{a}_{i}^{\mathrm{H}} \mathbf{x}\right|^{2}+b_{i}\right)\right] .
$$

In the works of [31], [32], [33], [34], [35], [36], various authors have considered data models similar to (5) for phaseretrieval for the case of background signal $b_{i}=0$. However, background signal is rarely zero in real world applications. The authors of [36] have proposed a variational model based on Total Variation (TV) regularization for phase-retrieval problem and an algorithm based on ADMM to solve a problem similar to (6). The authors of [37] have recently proposed three phaseretrieval algorithms for solving (6); the three algorithms they proposed are WF, MM and ADMM. In the WF algorithm for Poisson phase-retrieval, instead of using a heuristic step size, the authors have proposed a step size based on the observed Fisher information that can be computed without any tuning parameter. In their MM based algorithm, quadratic majorizer using improved curvature was used; however, this algorithm does not work for cases where the background signal $b_{i}=0$. To handle such cases, an algorithm based on ADMM was introduced which is somewhat similar to the ADMM based phase-retrieval algorithm proposed in [36].

In this paper, we propose a new algorithm for Poisson phase-retrieval problem which is based on MM framework and it is different from the MM based algorithm proposed in [37]. The proposed algorithm introduces an auxiliary variable into the original minimization problem and converts it into a saddle-point minimax problem by invoking Fenchel dual representation of the log term in the original cost function. The modified problem is then solved using MM framework by proposing surrogate functions over both the primal and dual variables to obtain a saddle-point. The resulting double loop MM algorithm is named as Primal-Dual MajorizationMinimization (PDMM) algorithm and is referred by this name henceforth. The MM-based algorithm proposed in [37] does not work when the background signal $b_{i}=0$, whereas PDMM effectively handles both $b_{i}>0$ and $b_{i}=0$ cases. Moreover, the surrogate function employed in PDMM is numerically shown to be tighter to the objective function in (6) than the quadratic surrogate function proposed in [37]. (Please refer to Fig. 1 and the related discussion given in section III-B).

The main contributions of this paper can be summarized as follows:

(i) We have devised a novel primal-dual MM algorithm which is different and efficient from the conventional MM method (which is restrictive as it is applicable only for the case $b_{i}>0$ ) for Poisson phase-retrieval problem.

(ii) We present the computationally efficient implementation of the proposed algorithm requiring only simple matrixvector product and Hadamard product of vectors.

(iii) We discuss the extension of our PDMM algorithm for the regularized Poisson phase-retrieval problem with the choice of regularizer $r(\mathbf{x})=\|\mathbf{T x}\|_{1}$, where unlike the restriction needed in the literature, the matrix $\mathbf{T}$ may not be proximal friendly. 
(iv) We discuss the proof of convergence of the proposed algorithm and show that the algorithm always converges to a stationary point of the Poisson likelihood function.

(v) We present several numerical simulation results (for both one-dimensional and two-dimensional signals) under different experimental settings and make performance comparisons with previously proposed algorithms (WF, conventional MM, and ADMM).

The rest of the paper is organized as follows: Section II formulates the minimization problem for Poisson phase-retrieval data model. Section III gives a brief overview of the MM framework, introduces the proposed algorithm, and discusses the computational complexity and the proof of convergence. Section IV gives the numerical simulation details and results under various experimental settings and comparison with the results of state-of-the-art algorithms. Finally, section V concludes the paper.

Notation : Bold upper case letters (e.g., A , C) denotes matrices while bold lower case letters (e.g., x , y) denotes column vectors. Italics (e.g., $c, d$ ) denotes scalars. For a vector $\mathbf{x},|\mathbf{x}|$ denotes element-wise magnitude, $x_{i}$ denotes the $i^{\text {th }}$ element of the vector, $\|\mathbf{x}\|_{2}$ denotes the Euclidean norm and $\mathbf{x} \circ \mathbf{y}$ denotes the Hadamard product with vector $\mathbf{y}$. The superscript $(.)^{*}$ denotes the conjugate, and the superscript $(.)^{\mathrm{H}}$, $(.)^{\mathrm{T}}$ and $(.)^{\dagger}$ denotes the conjugate transpose, transpose and the pseudo-inverse of a matrix respectively. For a complex number $x, \operatorname{Re}(x)$ and $\operatorname{Im}(x)$ denotes the real and imaginary parts respectively. I denotes the Identity matrix. The symbol $\operatorname{diag}(\mathbf{x})$ denotes the diagonal matrix formed by the elements of vector $\mathbf{x}$ as the principal diagonal and $\operatorname{diag}(\mathbf{X})$ denotes the column vector formed by the diagonal elements of matrix $\mathbf{X}$. The symbol trace $(\mathbf{X})$ denotes the trace of the matrix $\mathbf{X}$. The subscript $\mathbf{x}_{t}$ denotes the vector $\mathbf{x}$ at the $t^{\text {th }}$ iteration, and the vector $\mathbf{x}^{\text {opt }}$ denotes the optimal value of $\mathbf{x}$.

\section{PROBLEM FORMULATION}

In the case of Poisson data model, $y_{i} \sim$ Poisson $\left(\left|\mathbf{a}_{i}^{\mathrm{H}} \mathbf{x}\right|^{2}+b_{i}\right), i=1, \ldots, N$, the $\mathrm{ML}$ estimation corresponds to solving the following minimization problem:

$$
\min _{\mathbf{x}}\left\{f(\mathbf{x}) \triangleq \sum_{i=1}^{N}\left[\left|\mathbf{a}_{i}^{\mathrm{H}} \mathbf{x}\right|^{2}+b_{i}-y_{i} \log \left(\left|\mathbf{a}_{i}^{\mathrm{H}} \mathbf{x}\right|^{2}+b_{i}\right)\right]\right\} .
$$

The function $f(\mathbf{x})$ is non-convex due to the presence of quadratic term inside $-\log ($.$) , which also makes it a chal-$ lenging optimization problem to solve. To devise the proposed algorithm, we use the Fenchel representation of the log function [38] given as:

$$
-\log (u)=\max _{z \geq 0} \log (z)-z u+1
$$

Using the above mentioned representation, we get the following saddle-point formulation which is equivalent to the original optimization problem:

$$
\min _{\mathbf{x}} \max _{\mathbf{z} \geq 0} \sum_{i=1}^{N}\left[\left|\mathbf{a}_{i}^{\mathrm{H}} \mathbf{x}\right|^{2}+b_{i}+y_{i} \log \left(z_{i}\right)-z_{i}\left(\left|\mathbf{a}_{i}^{\mathrm{H}} \mathbf{x}\right|^{2}+b_{i}\right)\right],
$$

where $\mathbf{z}$ denotes the vector containing the elements $\left\{z_{i}\right\}$. The equivalence of (7) and (9) can be proved as follows. Consider the inner maximization problem over $z_{i}$ :

$$
\max _{\mathbf{z} \geq 0} \sum_{i=1}^{N}\left[y_{i} \log \left(z_{i}\right)-z_{i}\left(\left|\mathbf{a}_{i}^{\mathrm{H}} \mathbf{x}\right|^{2}+b_{i}\right)\right] .
$$

The above problem is separable in $z_{i}$. Therefore, a generic problem in $z$ (independent of index " $i$ ") can be written as

$$
\max _{z \geq 0} y \log (z)-z\left(\left|\mathbf{a}^{\mathrm{H}} \mathbf{x}\right|^{2}+b\right) .
$$

Writing the KKT conditions for the above problem and solving for $z$, we get

$$
z^{\mathrm{opt}}=\frac{y}{\left|\mathbf{a}^{\mathrm{H}} \mathbf{x}\right|^{2}+b} .
$$

Putting the optimal value $z_{i}^{\text {opt }}$ back in (9) we get the following minimization problem

$$
\begin{array}{r}
\min _{\mathbf{x}} \sum_{i=1}^{N}\left[\left|\mathbf{a}_{i}^{\mathrm{H}} \mathbf{x}\right|^{2}+b_{i}+y_{i} \log \left(\frac{y_{i}}{\left|\mathbf{a}_{i}^{\mathrm{H}} \mathbf{x}\right|^{2}+b_{i}}\right)\right. \\
\left.-\frac{y_{i}}{\left|\mathbf{a}_{i}^{\mathrm{H}} \mathbf{x}\right|^{2}+b_{i}}\left(\left|\mathbf{a}_{i}^{\mathrm{H}} \mathbf{x}\right|^{2}+b_{i}\right)\right] .
\end{array}
$$

Simplifying the above problem yields:

$$
\min _{\mathbf{x}} \sum_{i=1}^{N}\left[\left|\mathbf{a}_{i}^{\mathrm{H}} \mathbf{x}\right|^{2}+b_{i}-y_{i} \log \left(\left|\mathbf{a}_{i}^{\mathrm{H}} \mathbf{x}\right|^{2}+b_{i}\right)\right],
$$

which is same as problem (7). Thus the proof of the equivalence between (7) and (9) is established.

By introducing an auxiliary variable $z_{i}$ (which can also be viewed as a dual variable) in the original problem, the minimization problem is converted into a minimax problem. A solution ( $\mathbf{x}^{\mathrm{opt}}, \mathbf{z}^{\mathrm{opt}}$ ) of problem (9) would be a saddle-point for the minimax problem and hence the reformulation in (9) can also be termed as a saddle-point problem. Thus the crux of the proposed algorithm is to utilize Fenchel representation to remove quadratic term inside the $\log ($.$) and solve the$ equivalent saddle-point problem (9) instead of the original problem (7) to arrive at an optimal solution for $\mathbf{x}$.

The reader may doubt how the reformulation in (9), which looks complicated (as it is a minimax problem) than the original problem in (7), would be helpful, but in the next section we will show clearly that it is indeed easy to work with (9) and an MM algorithm can be devised. Moreover, the surrogate functions devised in this case result in a much tighter approximation to the original objective function in (7) than the surrogate function employed by the MM algorithm developed in [37].

\section{PDMM: THE PROPOSED PHASE-RETRIEVAL ALGORITHM}

This section first gives a brief overview of the MM framework which forms the backbone of the proposed algorithm. The proposed algorithms for the unregularized and regularized phase-retrieval problem are then explained later in detail. The section ends with a discussion on computational complexity and the proof of convergence of the proposed algorithm. 


\section{A. MM Framework [39]}

For cases where the original minimization problem has a complicated form, as in (7) and (9) (in case of (9), the objective of the minimization problem also involves maximization operation), MM exploits the problem structure and devises a problem-driven algorithm. It works in two steps. The first step is the majorization step, where a surrogate function which globally upperbounds the objective function, with their difference minimized at the current point is constructed. The second step is the minimization step, where the surrogate function obtained in the first step is minimized.

Consider the following minimization problem:

$$
\begin{aligned}
& \min _{\mathbf{x}} f(\mathbf{x}) \\
& \text { subject to } \mathbf{x} \in \mathcal{X},
\end{aligned}
$$

where $\mathcal{X}$ is a non-empty closed set in $\mathbb{R}^{n} / \mathbb{C}^{n}$, and $f(\mathbf{x})$ is a continuous function. It is assumed that $f(\mathbf{x})$ goes to infinity when $\|\mathbf{x}\|_{2} \rightarrow+\infty$. In MM, a point $\mathbf{x}_{0} \in \mathcal{X}$ is initialized and a series of feasible points $\mathbf{x}_{t}$ is generated. In the first step, a surrogate function $g\left(\mathbf{x} \mid \mathbf{x}_{t}\right)$ at $\mathbf{x}_{t}$ is constructed satisfying the following properties:

$$
g\left(\mathbf{x} \mid \mathbf{x}_{t}\right) \geq f(\mathbf{x}), \forall \mathbf{x} \in \mathcal{X}
$$

and

$$
g\left(\mathbf{x}_{t} \mid \mathbf{x}_{t}\right)=f\left(\mathbf{x}_{t}\right)
$$

In the second step which is the minimization step, $\mathbf{x}$ is updated as

$$
\mathbf{x}_{t+1}=\arg \min _{\mathbf{x} \in \mathcal{X}} g\left(\mathbf{x} \mid \mathbf{x}_{t}\right)
$$

From (16), (17) and (18), the following inequality is deduced:

$$
f\left(\mathbf{x}_{t+1}\right) \leq g\left(\mathbf{x}_{t+1} \mid \mathbf{x}_{t}\right) \leq g\left(\mathbf{x}_{t} \mid \mathbf{x}_{t}\right)=f\left(\mathbf{x}_{t}\right)
$$

which shows that the sequence $\left(f\left(\mathbf{x}_{t}\right)\right)$ is non-increasing. Thus, the objective function decreases monotonically using MM. The success of MM lies in the appropriate formulation of surrogate function. If the surrogate function is smooth and convex, and separable in variables, its minimization becomes efficient and scalable, leading to algorithms that can be easily implemented. However, while formulating a surrogate function, there is a trade-off between faster convergence and low computational cost/memory requirements per iteration. For faster convergence, the surrogate function should follow the shape of the objective function as close as possible. Whereas, to keep the computational complexity low, the surrogate function should be simple to minimize. Balancing this trade-off is the key to the successful implementation of MM.

Here we would like to mention that the general discussion for the MM framework presented in this subsection is for a minimization problem (as in (15)), however our problem of interest (9) is a minimax problem. The next subsection explains how the above mentioned steps of the MM can be adapted for a minimax problem.
B. Primal-Dual Majorization-Minimization (PDMM) Algorithm

Let us start with the cost function (only over $\mathbf{x}$ ) in (9),

$$
\begin{aligned}
f(\mathbf{x}) \triangleq \max _{\mathbf{z} \geq 0} h(\mathbf{x}, \mathbf{z}) \triangleq \max _{\mathbf{z} \geq 0} & \left(\mathbf{x}^{\mathrm{H}} \mathbf{A}^{\mathrm{H}} \mathbf{A} \mathbf{x}+\sum_{i=1}^{N} y_{i} \log \left(z_{i}\right)\right. \\
& \left.-\sum_{i=1}^{N} z_{i} \mathbf{x}^{\mathrm{H}} \mathbf{a}_{i} \mathbf{a}_{i}^{\mathrm{H}} \mathbf{x}-\sum_{i=1}^{N} z_{i} b_{i}\right) .
\end{aligned}
$$

Then the optimization problem given in (9) can be rewritten as:

$$
\min _{\mathbf{x}} f(\mathbf{x})
$$

The third term of $h(\mathbf{x}, \mathbf{z})$ in (20), i.e., $-\sum_{i=1}^{N} z_{i} \mathbf{x}^{\mathrm{H}} \mathbf{a}_{i} \mathbf{a}_{i}^{\mathrm{H}} \mathbf{x}$ is a concave function in $\mathbf{x}$ (for any $z_{i}$ ). Thus, we can use first-order Taylor's expansion to upperbound the concave term, which will be discussed shortly.

Let the third term of $h(\mathbf{x}, \mathbf{z})$ be denoted as:

$$
h_{3}(\mathbf{x}, \mathbf{z}) \triangleq-\sum_{i=1}^{N} z_{i} \mathbf{x}^{\mathrm{H}} \mathbf{a}_{i} \mathbf{a}_{i}^{\mathrm{H}} \mathbf{x},
$$

which we can also express as follows:

$$
h_{3}(\mathbf{x}, \mathbf{z})=\sum_{i=1}^{N} z_{i} \tilde{h}_{3 i}(\mathbf{x}),
$$

where $\tilde{h}_{3 i}(\mathbf{x})=-\mathbf{x}^{\mathrm{H}} \mathbf{a}_{i} \mathbf{a}_{i}^{\mathrm{H}} \mathbf{x}$, which is clearly a concave function in $\mathbf{x}$. Using the first-order Taylor's expansion for $\tilde{h}_{3 i}(\mathbf{x})$ at any $\mathbf{x}=\mathbf{x}_{t}$,

$$
\tilde{h}_{3 i}(\mathbf{x}) \leq \tilde{h}_{3 i}\left(\mathbf{x}_{t}\right)+\operatorname{Re}\left(\nabla \tilde{h}_{3 i}\left(\mathbf{x}_{t}\right)^{\mathrm{H}}\left(\mathbf{x}-\mathbf{x}_{t}\right)\right),
$$

we get the following MM inequality:

$$
-\mathbf{x}^{\mathrm{H}} \mathbf{a}_{i} \mathbf{a}_{i}^{\mathrm{H}} \mathbf{x} \leq \mathbf{x}_{t}^{\mathrm{H}} \mathbf{a}_{i} \mathbf{a}_{i}^{\mathrm{H}} \mathbf{x}_{t}-2 \operatorname{Re}\left(\mathbf{x}_{t}^{\mathrm{H}} \mathbf{a}_{i} \mathbf{a}_{i}^{\mathrm{H}} \mathbf{x}\right),
$$

where the equality is achieved when $\mathbf{x}=\mathbf{x}_{t}$. Thus, using (25) $h_{3}(\mathbf{x}, \mathbf{z})$ can be upperbounded as

$-\sum_{i=1}^{N} z_{i} \mathbf{x}^{\mathrm{H}} \mathbf{a}_{i} \mathbf{a}_{i}^{\mathrm{H}} \mathbf{x} \leq \sum_{i=1}^{N} z_{i} \mathbf{x}_{t}^{\mathrm{H}} \mathbf{a}_{i} \mathbf{a}_{i}^{\mathrm{H}} \mathbf{x}_{t}-2 \sum_{i=1}^{N} \operatorname{Re}\left(z_{i} \mathbf{x}_{t}^{\mathrm{H}} \mathbf{a}_{i} \mathbf{a}_{i}^{\mathrm{H}} \mathbf{x}\right)$.

Using (26), the objective function in (20) can be upperbounded as

$$
\begin{aligned}
f(\mathbf{x})= & \max _{\mathbf{z} \geq 0} h(\mathbf{x}, \mathbf{z}) \leq \max _{\mathbf{z} \geq 0}\left(\mathbf{x}^{\mathrm{H}} \mathbf{A}^{\mathrm{H}} \mathbf{A} \mathbf{x}-2 \sum_{i=1}^{N} \operatorname{Re}\left(z_{i} \mathbf{x}_{t}^{\mathrm{H}} \mathbf{a}_{i} \mathbf{a}_{i}^{\mathrm{H}} \mathbf{x}\right)\right. \\
& \left.+\sum_{i=1}^{N} z_{i} \mathbf{x}_{t}^{\mathrm{H}} \mathbf{a}_{i} \mathbf{a}_{i}^{\mathrm{H}} \mathbf{x}_{t}+\sum_{i=1}^{N} y_{i} \log \left(z_{i}\right)-\sum_{i=1}^{N} z_{i} b_{i}\right) \triangleq g_{f}\left(\mathbf{x} \mid \mathbf{x}_{t}\right) .
\end{aligned}
$$

Thus, we arrive at the following surrogate optimization problem:

$$
\begin{array}{r}
\min _{\mathbf{x}}\left\{g_{f}\left(\mathbf{x} \mid \mathbf{x}_{t}\right)=\max _{\mathbf{z} \geq 0}\left(\mathbf{x}^{\mathrm{H}} \mathbf{A}^{\mathrm{H}} \mathbf{A} \mathbf{x}+\sum_{i=1}^{N} y_{i} \log \left(z_{i}\right)-\sum_{i=1}^{N} z_{i} b_{i}\right.\right. \\
\left.\left.+\sum_{i=1}^{N} z_{i}\left|d_{i}\right|^{2}-2 \sum_{i=1}^{N} \operatorname{Re}\left(z_{i} d_{i}^{*} \mathbf{a}_{i}^{\mathrm{H}} \mathbf{x}\right)\right)\right\},
\end{array}
$$

where $d_{i} \triangleq \mathbf{a}_{i}^{\mathrm{H}} \mathbf{x}_{t}$. It can be noted that the above problem has an inner maximization problem in $\mathbf{z}$ and an outer maximization 
problem in $\mathbf{x}$. If we solve the inner maximization problem and substitute back the optimal maximizer $\mathbf{z}^{\mathrm{opt}}$, we get a minimization problem only in the primal variable $\mathbf{x}$, in which the objective (in $\mathbf{x}$ ) would be a MM surrogate for the objective in (7). In the following remark we will explain more on this aspect.

Remark 1. The inner maximization problem of (28) can be written as:

$$
\max _{\mathbf{z} \geq 0} \sum_{i=1}^{N}\left[y_{i} \log \left(z_{i}\right)-z_{i} b_{i}+z_{i}\left|d_{i}\right|^{2}-2 \operatorname{Re}\left(z_{i} d_{i}^{*} \mathbf{a}_{i}^{\mathrm{H}} \mathbf{x}\right)\right] .
$$

It is obvious from the objective in (29) that for any $i$, when $b_{i}-\left|d_{i}\right|^{2}+2 \operatorname{Re}\left(d_{i}^{*} \mathbf{a}_{i}^{\mathrm{H}} \mathbf{x}\right) \leq 0$, the optimization problem in (29) would be unbounded above, and when $b_{i}-\left|d_{i}\right|^{2}+$ $2 \operatorname{Re}\left(d_{i}^{*} \mathbf{a}_{i}^{\mathrm{H}} \mathbf{x}\right)>0$, the optimal value of $z_{i}$ would be

$$
z_{i}^{\mathrm{opt}}=\frac{y_{i}}{b_{i}-\left|d_{i}\right|^{2}+2 \operatorname{Re}\left(d_{i}^{*} \mathbf{a}_{i}^{\mathrm{H}} \mathbf{x}\right)} .
$$

Substituting back $z_{i}^{\text {opt }}$ in (28) (with the condition that $b_{i}-$ $\left.\left|d_{i}\right|^{2}+2 \operatorname{Re}\left(d_{i}^{*} \mathbf{a}_{i}^{\mathrm{H}} \mathbf{x}\right)>0 \forall i\right)$, we get the following surrogate problem of PDMM in primal variable $\mathrm{x}$ :

$$
\begin{array}{r}
\min _{\mathbf{x}}\left(\mathbf{x}^{\mathrm{H}} \mathbf{A}^{\mathrm{H}} \mathbf{A} \mathbf{x}-\sum_{i=1}^{N}\left(\frac{b_{i} y_{i}}{b_{i}-\left|d_{i}\right|^{2}+2 \operatorname{Re}\left(d_{i}^{*} \mathbf{a}_{i}^{\mathrm{H}} \mathbf{x}\right)}\right)\right. \\
+\sum_{i=1}^{N} y_{i} \log \left(\frac{y_{i}}{b_{i}-\left|d_{i}\right|^{2}+2 \operatorname{Re}\left(d_{i}^{*} \mathbf{a}_{i}^{\mathrm{H}} \mathbf{x}\right)}\right) \\
-2 \sum_{i=1}^{N} \operatorname{Re}\left(\frac{y_{i} d_{i}^{*} \mathbf{a}_{i}^{\mathrm{H}} \mathbf{x}}{b_{i}-\left|d_{i}\right|^{2}+2 \operatorname{Re}\left(d_{i}^{*} \mathbf{a}_{i}^{\mathrm{H}} \mathbf{x}\right)}\right) \\
\left.+\sum_{i=1}^{N}\left(\frac{y_{i}\left|d_{i}\right|^{2}}{b_{i}-\left|d_{i}\right|^{2}+2 \operatorname{Re}\left(d_{i}^{*} \mathbf{a}_{i}^{\mathrm{H}} \mathbf{x}\right)}\right)\right) \\
\text { subject to } b_{i}-\left|d_{i}\right|^{2}+2 \operatorname{Re}\left(d_{i}^{*} \mathbf{a}_{i}^{\mathrm{H}} \mathbf{x}\right)>0 \forall i
\end{array}
$$

The objective in (31) is a surrogate function for the original Poisson likelihood function in (7). For instance, consider a one-dimensional example with two data samples $(N=2)$, the elements of $\mathbf{A} \in \mathbb{R}^{1 \times 2}$ randomly generated, $b_{i}=0.1$, and $\mathbf{y}$ generated via the model in (5) with the choice of true $x$ $\left(x_{\text {true }}\right)$ fixed to 8 . Fig. 1 shows the plots of the objective in (7) around the neighbourhood of $x_{\text {true }}$, the objective of (31) satisfying the constraint, and also the "MM" surrogate function developed in [37] with the choice of $x_{t}=4$. From Fig. 1 it can be seen that both the function of (31) and the quadratic majorizer of [37] exactly match at $x=4$ (as required in MM), however the function in (31) closely follows the shape of the original Poisson likelihood function and hence is a tighter approximation to it than the one proposed in [37], and it is more likely to yield a faster converging algorithm if we solve (31), then iterate and so on. However, (31) does not have a closed form solution and requires a convex solver like CVX to solve it, so we proceed further by reformulating the minimax problem (28) into a maximin problem and look for other possibilities to solve the problem. Nonetheless, Fig.1 clearly shows that the surrogate constructed in PDMM is a tighter approximation to the original objective than the surrogate proposed in [37].

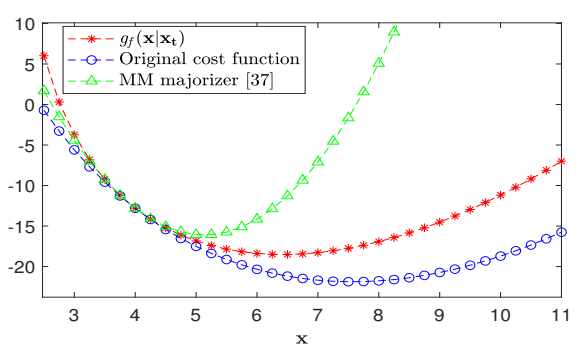

Fig. 1. A representative plot of majorizers for PDMM (satisfying the constraint in (31)) and MM [37] with $x_{t}=4$ for the non-convex Poisson log-likelihood function.

To proceed further, we first rewrite the optimization problem in (28) as:

$$
\begin{aligned}
\min _{\mathbf{x}} \max _{\mathbf{z} \geq 0} & \left(\mathbf{x}^{\mathrm{H}} \mathbf{A}^{\mathrm{H}} \mathbf{A} \mathbf{x}+\sum_{i=1}^{N} y_{i} \log \left(z_{i}\right)-\sum_{i=1}^{N} z_{i} b_{i}\right. \\
& \left.+\sum_{i=1}^{N} z_{i}\left|d_{i}\right|^{2}-2 \sum_{i=1}^{N} \operatorname{Re}\left(z_{i} d_{i}^{*} \mathbf{a}_{i}^{\mathrm{H}} \mathbf{x}\right)\right)
\end{aligned}
$$

The minimax problem in (32) can be reformulated as a maximin problem using minimax theorem [40] (which is briefly stated in the following lemma):

Lemma 2. Let $\mathcal{X} \subset \mathbb{R}^{n}$ and $\mathcal{Y} \subset \mathbb{R}^{m}$ be compact convex sets. If $f: \mathcal{X} \times \mathcal{Y} \rightarrow \mathbb{R}$ is a continuous function that is concave-convex, i.e., $f(., y): \mathcal{X} \rightarrow \mathbb{R}$ is concave for fixed $y$, and $f(x,):. \mathcal{Y} \rightarrow \mathbb{R}$ is convex for fixed $x$, then we have

$$
\max _{x \in \mathcal{X}} \min _{y \in \mathcal{Y}} f(x, y)=\min _{y \in \mathcal{Y}} \max _{x \in \mathcal{X}} f(x, y)
$$

Although the variable $\mathbf{x} \in \mathbb{C}^{K}$ is complex valued, if we rewrite (32) using a new variable $\tilde{\mathbf{x}} \triangleq[\operatorname{Re}(\mathbf{x}) ; \operatorname{Im}(\mathbf{x})] \in \mathbb{R}^{2 K}$, the objective function in (32) would still be a continuous function which is convex in $\tilde{\mathbf{x}}$ for fixed $\mathbf{z}$ and concave in $\mathbf{z}$ for fixed $\tilde{\mathbf{x}}$. Therefore Lemma 2 can be applied to the minimax problem in (32). Thus, swapping min and max in (28), we get

$$
\begin{array}{r}
\max _{\mathbf{z} \geq 0} \min _{\mathbf{x}}\left(\mathbf{x}^{\mathrm{H}} \mathbf{A}^{\mathrm{H}} \mathbf{A} \mathbf{x}-2\right. \\
\operatorname{Re}\left(\sum_{i=1}^{N}\left(z_{i} d_{i}^{*} \mathbf{a}_{i}^{\mathrm{H}} \mathbf{x}\right)\right)-\sum_{i=1}^{N} z_{i} b_{i} \\
\left.+\sum_{i=1}^{N} y_{i} \log \left(z_{i}\right)+\sum_{i=1}^{N} z_{i}\left|d_{i}\right|^{2}\right) .
\end{array}
$$

The maximin problem (33) has an inner minimization problem in $\mathbf{x}$ and an outer maximization problem in $\mathbf{z}$. We first solve the inner minimization problem which is given by:

$$
\min _{\mathbf{x}} \mathbf{x}^{\mathrm{H}} \mathbf{A}^{\mathrm{H}} \mathbf{A} \mathbf{x}-2 \operatorname{Re}\left(\sum_{i=1}^{N}\left(z_{i} d_{i}^{*} \mathbf{a}_{i}^{\mathrm{H}} \mathbf{x}\right)\right) \text {. }
$$

The minimizer over $\mathbf{x}$ is given as:

$$
\mathbf{x}_{t+1}=\left(\mathbf{A}^{\mathrm{H}} \mathbf{A}\right)^{-1} \mathbf{A}^{\mathrm{H}} \mathbf{D} \mathbf{z}
$$

where $\mathbf{D}=\operatorname{diag}(\mathbf{d})$ i.e. a diagonal matrix formed by setting elements of $\mathbf{d}$ as its principal diagonal. It is to be noted that $\left(\mathbf{A}^{\mathrm{H}} \mathbf{A}\right)^{-1} \mathbf{A}^{\mathrm{H}}$ is the pseudo-inverse of matrix $\mathbf{A}$ and hence (35) can be compactly written as:

$$
\mathbf{x}_{t+1}=\mathbf{A}^{\dagger}(\mathbf{d} \circ \mathbf{z})
$$


The pseudo-inverse $\mathbf{A}^{\dagger}$ is not iteration dependent and can be calculated and stored (for suitably small problems) once outside the iteration loops which will help us to implement the algorithm in an efficient manner. By substituting back $\mathbf{x}_{t+1}$ in (33) we get

$$
\begin{array}{r}
\max _{\mathbf{z} \geq 0}\left(-\mathbf{z}^{\mathrm{H}} \mathbf{D}^{\mathrm{H}} \mathbf{A}\left(\mathbf{A}^{\mathrm{H}} \mathbf{A}\right)^{-1} \mathbf{A}^{\mathrm{H}} \mathbf{D} \mathbf{z}+\sum_{i=1}^{N} y_{i} \log \left(z_{i}\right)\right. \\
\left.-\sum_{i=1}^{N} z_{i} b_{i}+\sum_{i=1}^{N} z_{i}\left|d_{i}\right|^{2}\right) .
\end{array}
$$

It is to be noted here that although the vector $\mathbf{z}$ is realvalued, we use $\mathbf{z}^{\mathrm{H}}$ to denote its transpose for consistency in the notation. The maximization problem (37) is first converted into the following minimization problem for convenience:

$$
\begin{array}{r}
\min _{\mathbf{z} \geq 0} \mathbf{z}^{\mathrm{H}} \mathbf{D}^{\mathrm{H}} \mathbf{A}\left(\mathbf{A}^{\mathrm{H}} \mathbf{A}\right)^{-1} \\
\mathbf{A}^{\mathrm{H}} \mathbf{D} \mathbf{z}-\sum_{i=1}^{N} y_{i} \log \left(z_{i}\right) \\
+\sum_{i=1}^{N} z_{i} b_{i}-\sum_{i=1}^{N} z_{i}\left|d_{i}\right|^{2}
\end{array}
$$

The above mentioned problem is a convex minimization problem in $\left\{z_{i}\right\}^{\prime} s$ and can be solved directly using interior point solver like CVX [41]. However, it would be computationally inefficient to use CVX (especially for large dimensional problem setting). Therefore, in the following we explore the use of MM (over variables $z_{i}$ ) again to find the global minimizer of the problem in (38). We construct a surrogate function for the objective in (38) at some given $\mathbf{z}_{k}$ and minimize the surrogate iteratively to arrive at the optimal minimizer of (38).

Let $\mathbf{P} \triangleq \mathbf{A}\left(\mathbf{A}^{\mathrm{H}} \mathbf{A}\right)^{-1} \mathbf{A}^{\mathrm{H}}$ and $\mathbf{I}$ denote an identity matrix of dimension $N \times N$. The minimization problem (38) can be rewritten as:

$$
\begin{array}{r}
\min _{\mathbf{z} \geq 0}\left(\mathbf{z}^{\mathrm{H}} \mathbf{D}^{\mathrm{H}}(\mathbf{P}-\mathbf{I}) \mathbf{D} \mathbf{z}+\mathbf{z}^{\mathrm{H}} \mathbf{D}^{\mathrm{H}} \mathbf{D} \mathbf{z}+\sum_{i=1}^{N} z_{i} b_{i}\right. \\
\left.-\sum_{i=1}^{N} y_{i} \log \left(z_{i}\right)-\sum_{i=1}^{N} z_{i}\left|d_{i}\right|^{2}\right) .
\end{array}
$$

Remark 3. It is to be noted that $\mathbf{P}$ is a projection matrix onto the column space of matrix $\mathbf{A}$. The matrix $\mathbf{I}-\mathbf{P}$ is then the projection matrix onto its orthogonal complement or null space of $\mathbf{A}^{\mathrm{H}}$. Therefore, matrices $\mathbf{P}$ and $\mathbf{I}-\mathbf{P}$ are Hermitian, positive semi-definite, and have a maximum eigenvalue equal to 1. The matrix $\mathbf{P}-\mathbf{I}$ is therefore a negative semi-definite matrix which makes the first term in problem (39) concave.

The concave term in (39) is linearized (via first-order Taylor series expansion, similar to (24), at $\mathbf{z}=\mathbf{z}_{k}$ ) and an upperbound is obtained, using which we arrive at the following surrogate problem:

$$
\begin{array}{r}
\min _{\mathbf{z} \geq 0}\left(2 \operatorname{Re}\left(\mathbf{z}^{\mathrm{H}} \mathbf{D}^{\mathrm{H}}(\mathbf{P}-\mathbf{I}) \mathbf{D} \mathbf{z}_{k}\right)+\mathbf{z}^{\mathrm{H}} \mathbf{D}^{\mathrm{H}} \mathbf{D} \mathbf{z}+\sum_{i=1}^{N} z_{i} b_{i}\right. \\
\left.-\sum_{i=1}^{N} y_{i} \log \left(z_{i}\right)-\sum_{i=1}^{N} z_{i}\left|d_{i}\right|^{2}\right) .
\end{array}
$$

With $\mathbf{c} \triangleq 2 \operatorname{Re}\left(\mathbf{D}^{\mathrm{H}}(\mathbf{P}-\mathbf{I}) \mathbf{D} \mathbf{z}_{k}\right)$ and $h_{i} \triangleq\left|d_{i}\right|^{2}$, the above mentioned problem becomes:

$$
\min _{\mathbf{z} \geq 0} \sum_{i=1}^{N}\left[c_{i} z_{i}+h_{i} z_{i}^{2}+b_{i} z_{i}-y_{i} \log \left(z_{i}\right)-h_{i} z_{i}\right] .
$$

which is separable in $z_{i}$. Thus, a generic problem (without the index $i$ ) can be written as

$$
\min _{z \geq 0} c z+h z^{2}+b z-y \log (z)-h z .
$$

The Karush-Kuhn-Tucker (KKT) condition for (42) will be

$$
c+2 h z+b-\frac{y}{z}-h=0,
$$

which can also be written as:

$$
c z+2 h z^{2}+b z-y-h z=0 .
$$

Solving (44), we arrive at the optimal solution over $z$ which will serve as the next iterate:

$$
z_{k+1}= \begin{cases}\frac{-(b+c-h)+\sqrt{(b+c-h)^{2}+8 h y}}{4 h} & \text { if } h \neq 0 \\ \frac{y}{b+c} & \text { if } h=0\end{cases}
$$

The pseudo code for the PDMM algorithm obtained is given in the Algorithm Table 1.

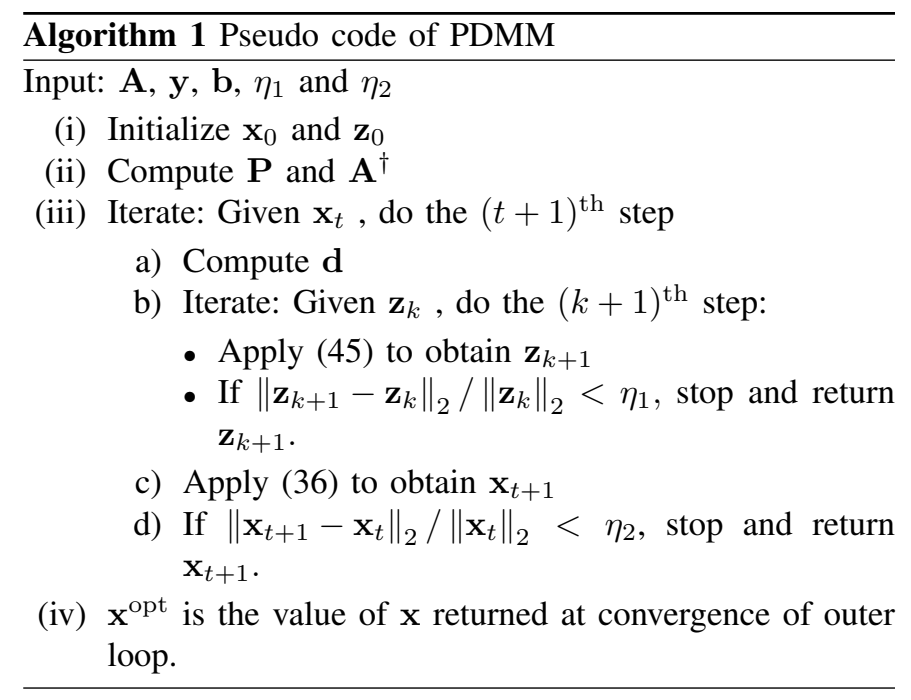

Remark 4. The MM iterations over the variable $\mathbf{z}$ need not necessarily be run till convergence. The inner loop iterations can be stopped adaptively by computing $\mathbf{x}_{t+1}$ in (36) using the recent value of $\mathbf{z}$ and checking if $f\left(\mathbf{x}_{t+1}\right) \leq f\left(\mathbf{x}_{t}\right)$.

\section{PDMM for regularized Poisson phase-retrieval problem}

The previous subsection describes the algorithm for minimizing the unregularized problem, which is an ill-posed problem, especially when $N<K$, so for the stable recovery of the original signal, one requires some prior knowledge on the signal. One of the ways to deal with this problem is to impose sparsity (which the underlying signal would also possess) by incorporating an additional term (regularizer or penalty) in the cost function [42], [43], [44]. In this subsection, we adapt the derivation for the regularized version of the Poisson loglikelihood problem using the $\ell_{1}$ norm regularizer which is well known to produce a sparse solution. 
Let $r(\mathbf{x})$ be the regularizer, then the regularized problem would be:

$$
\phi(\mathbf{x})=f(\mathbf{x})+\lambda r(\mathbf{x})
$$

where $\lambda$ is the regularization parameter and $r(\mathbf{x})=\|\mathbf{T} \mathbf{x}\|_{1}$. The different choices for $\mathbf{T}$ matrix can be Identity matrix, Orthogonal Discrete Wavelet Transform (ODWT) matrix or the finite-difference matrix used in Total variation (TV) regularization.

Using $r(\mathbf{x})=\|\mathbf{T} \mathbf{x}\|_{1}$, the saddle point representation of the regularized problem can be written as:

$$
\begin{array}{r}
\min _{\mathbf{x}} \max _{\mathbf{z} \geq 0}\left(\mathbf{x}^{\mathrm{H}} \mathbf{A}^{\mathrm{H}} \mathbf{A} \mathbf{x}-\sum_{i=1}^{N} z_{i} \mathbf{x}^{\mathrm{H}} \mathbf{a}_{i} \mathbf{a}_{i}^{\mathrm{H}} \mathbf{x}+\sum_{i=1}^{N} y_{i} \log \left(z_{i}\right)\right. \\
\left.-\sum_{i=1}^{N} z_{i} b_{i}+\lambda\|\mathbf{T} \mathbf{x}\|_{1}\right) .
\end{array}
$$

Now, introducing one more auxiliary variable $w_{l}$ 's to tackle the regularization term, we get the following equivalent problem:

$$
\begin{array}{r}
\min _{\mathbf{x}} \max _{\substack{\mathbf{z} \geq 0,\left|w_{l}\right| \leq 1}}\left(\mathbf{x}^{\mathrm{H}} \mathbf{A}^{\mathrm{H}} \mathbf{A} \mathbf{x}-\sum_{i=1}^{N} z_{i} \mathbf{x}^{\mathrm{H}} \mathbf{a}_{i} \mathbf{a}_{i}^{\mathrm{H}} \mathbf{x}+\sum_{i=1}^{N} y_{i} \log \left(z_{i}\right)\right. \\
\left.-\sum_{i=1}^{N} z_{i} b_{i}+\lambda \operatorname{Re}\left(\mathbf{w}^{\mathrm{H}} \mathbf{T} \mathbf{x}\right)\right),
\end{array}
$$

where $\mathbf{w}$ denotes the column vector containing $\left\{w_{l}\right\}_{l=1}^{K-1}$. It is easy to verify that if we compute the maximizer over $w_{l}^{\prime} s$ and substitute back the maximizer in the objective function in (48), we will obtain the problem in (47). Following the similar procedure as in the unregularized case, linearizing the concave term $-\sum_{i=1}^{N} z_{i} \mathbf{x}^{\mathrm{H}} \mathbf{a}_{i} \mathbf{a}_{i}^{\mathrm{H}} \mathbf{x}$ using first order Taylor expansion as given in (24), we get the following surrogate problem.

$$
\begin{array}{r}
\min _{\mathbf{x}} \max _{\substack{\mathbf{z} \geq 0 . \\
\left|w_{l}\right| \leq 1}}\left(\mathbf{x}^{\mathrm{H}} \mathbf{A}^{\mathrm{H}} \mathbf{A} \mathbf{x}-2 \operatorname{Re}\left(\sum_{i=1}^{N} z_{i} d_{i}^{*} \mathbf{a}_{i}^{\mathrm{H}} \mathbf{x}\right)+\sum_{i=1}^{N}\left|d_{i}\right|^{2} z_{i}\right. \\
\left.+\sum_{i=1}^{N} y_{i} \log \left(z_{i}\right)-\sum_{i=1}^{N} z_{i} b_{i}+\lambda \operatorname{Re}\left(\mathbf{w}^{\mathrm{H}} \mathbf{T} \mathbf{x}\right)\right) .
\end{array}
$$

Since the above mentioned problem is convex in $\mathrm{x}$ for fixed $\mathbf{z}$ and $\mathbf{w}$, concave in $\mathbf{z}$ for fixed $\mathbf{x}$ and $\mathbf{w}$, and linear in $\mathbf{w}$ for fixed $\mathbf{x}$ and $\mathbf{z}$, the minmax theorem stated in Lemma 2 can be applied on it in two steps. Swapping the $\min _{\mathbf{x}}$ and $\max _{\mathbf{z} \geq 0}$ terms, we get the intermediate problem:

$$
\begin{array}{r}
\max _{\mathbf{z} \geq 0} \min _{\mathbf{x}} \max _{w_{l} \leq 1}\left(\mathbf{x}^{\mathrm{H}} \mathbf{A}^{\mathrm{H}} \mathbf{A} \mathbf{x}-2 \operatorname{Re}\left(\sum_{i=1}^{N} z_{i} d_{i}^{*} \mathbf{a}_{i}^{\mathrm{H}} \mathbf{x}\right)+\sum_{i=1}^{N}\left|d_{i}\right|^{2} z_{i}\right. \\
\left.+\sum_{i=1}^{N} y_{i} \log \left(z_{i}\right)-\sum_{i=1}^{N} z_{i} b_{i}+\lambda \operatorname{Re}\left(\mathbf{w}^{\mathrm{H}} \mathbf{T} \mathbf{x}\right)\right) .
\end{array}
$$

In the second step, we swap $\min _{\mathbf{x}}$ and $\max _{w_{l} \leq 1}$ to get the following maximin optimization problem:

$$
\begin{array}{r}
\max _{\substack{\mathbf{z} \geq 0,\left|w_{l}\right| \leq 1}} \min _{\mathbf{x}}\left(\mathbf{x}^{\mathrm{H}} \mathbf{A}^{\mathrm{H}} \mathbf{A} \mathbf{x}-2 \operatorname{Re}\left(\sum_{i=1}^{N} z_{i} d_{i}^{*} \mathbf{a}_{i}^{\mathrm{H}} \mathbf{x}\right)+\sum_{i=1}^{N}\left|d_{i}\right|^{2} z_{i}\right. \\
\left.+\sum_{i=1}^{N} y_{i} \log \left(z_{i}\right)-\sum_{i=1}^{N} z_{i} b_{i}+\lambda \operatorname{Re}\left(\mathbf{w}^{\mathrm{H}} \mathbf{T} \mathbf{x}\right)\right) .
\end{array}
$$

Problem (51) has an inner maximization problem in $\mathbf{x}$ and an outer maximization problem in $\mathbf{z}$ and $\mathbf{w}$. We first solve the inner minimization problem which is given by:

$$
\min _{\mathbf{x}} \mathbf{x}^{\mathrm{H}} \mathbf{A}^{\mathrm{H}} \mathbf{A} \mathbf{x}-2 \operatorname{Re}\left(\sum_{\mathrm{i}=1}^{\mathrm{N}} \mathrm{z}_{\mathrm{i}} \mathrm{d}_{\mathrm{i}}^{*} \mathbf{a}_{\mathrm{i}}^{\mathrm{H}} \mathbf{x}\right)+\lambda \operatorname{Re}\left(\mathbf{w}^{\mathrm{H}} \mathbf{T} \mathbf{x}\right) .
$$

The optimal solution for $\mathbf{x}$ would be

$$
\mathbf{x}_{t+1}=\left(\mathbf{A}^{\mathrm{H}} \mathbf{A}\right)^{-1}\left(\mathbf{A}^{\mathrm{H}} \mathbf{D} \mathbf{z}-\frac{\lambda}{2} \mathbf{T}^{\mathrm{H}} \mathbf{w}\right),
$$

which can also be written as

$$
\mathbf{x}_{t+1}=\mathbf{A}^{\dagger}(\mathbf{d} \circ \mathbf{z})-\frac{\lambda}{2}\left(\mathbf{A}^{\mathrm{H}} \mathbf{A}\right)^{-1}\left(\mathbf{T}^{\mathrm{H}} \mathbf{w}\right) .
$$

The matrices $\mathbf{A}^{\dagger}$ and $\left(\mathbf{A} \mathbf{A}^{\mathrm{H}}\right)^{-1} \mathbf{T}^{\mathrm{H}}$ in (54) can be precomputed and stored since they are not iteration dependent. Substituting back $\mathbf{x}_{t+1}$ in (51) gives the following maximization problem:

$$
\begin{array}{r}
\max _{\substack{\mathbf{z} \geq 0,\left|w_{l}\right| \leq 1}}\left(-\mathbf{z}^{\mathrm{H}} \mathbf{D}^{\mathrm{H}} \mathbf{A}\left(\mathbf{A}^{\mathrm{H}} \mathbf{A}\right)^{-1} \mathbf{A}^{\mathrm{H}} \mathbf{D} \mathbf{z}+\sum_{i=1}^{N}\left|d_{i}\right|^{2} z_{i}+\sum_{i=1}^{N} y_{i} \log \left(z_{i}\right)\right. \\
+\frac{\lambda}{2} \mathbf{z}^{\mathrm{H}} \mathbf{D}^{\mathrm{H}} \mathbf{A}\left(\mathbf{A}^{\mathrm{H}} \mathbf{A}\right)^{-1} \mathbf{T}^{\mathrm{H}} \mathbf{w}+\frac{\lambda}{2} \mathbf{w}^{\mathrm{H}} \mathbf{T}\left(\mathbf{A}^{\mathrm{H}} \mathbf{A}\right)^{-1} \mathbf{A}^{\mathrm{H}} \mathbf{D} \mathbf{z} \\
\left.-\frac{\lambda^{2}}{4} \mathbf{w}^{\mathrm{H}} \mathbf{T}\left(\mathbf{A}^{\mathrm{H}} \mathbf{A}\right)^{-1} \mathbf{T}^{\mathrm{H}} \mathbf{w}-\sum_{i=1}^{N} z_{i} b_{i}\right) .
\end{array}
$$

We first rewrite the above problem into a minimization problem for convenience.

$$
\begin{array}{r}
\min _{\substack{\mathbf{z} \geq 0,\left|w_{l}\right| \leq 1}}\left(\mathbf{z}^{\mathrm{H}} \mathbf{D}^{\mathrm{H}} \mathbf{A}\left(\mathbf{A}^{\mathrm{H}} \mathbf{A}\right)^{-1} \mathbf{A}^{\mathrm{H}} \mathbf{D} \mathbf{z}-\sum_{i=1}^{N}\left|d_{i}\right|^{2} z_{i}-\sum_{i=1}^{N} y_{i} \log \left(z_{i}\right)\right. \\
+\sum_{i=1}^{N} z_{i} b_{i}-\lambda \operatorname{Re}\left(\mathbf{z}^{\mathrm{H}} \mathbf{D}^{\mathrm{H}} \mathbf{A}\left(\mathbf{A}^{\mathrm{H}} \mathbf{A}\right)^{-1} \mathbf{T}^{\mathrm{H}} \mathbf{w}\right) \\
\left.+\frac{\lambda^{2}}{4} \mathbf{w}^{\mathrm{H}} \mathbf{T}\left(\mathbf{A}^{\mathrm{H}} \mathbf{A}\right)^{-1} \mathbf{T}^{\mathrm{H}} \mathbf{w}\right) .
\end{array}
$$

The above problem can be reformulated as a jointly convex problem in $\mathbf{w}$ and $\mathbf{z}$ and can be solved using an interior point solver. However, similar to the previous sub-section, we proceed further to solve for $\mathbf{w}$ and $\mathbf{z}$ iteratively using MM. With $\mathbf{X} \triangleq\left(\mathbf{A}^{\mathrm{H}} \mathbf{A}\right)^{-1} \mathbf{T}^{\mathrm{H}}$ and $e$ denoting the maximum eigenvalue of $\mathbf{X}$ (which can be pre-computed and stored as it is not iteration dependent), problem (56) can be rewritten as:

$$
\begin{array}{r}
\max _{\substack{\mathbf{z} \geq 0,\left|w_{l}\right| \leq 1}}\left(\mathbf{z}^{\mathrm{H}} \mathbf{D}^{\mathrm{H}}(\mathbf{P}-\mathbf{I}) \mathbf{D} \mathbf{z}+\mathbf{z}^{\mathrm{H}} \mathbf{D}^{\mathrm{H}} \mathbf{D} \mathbf{z}+\sum_{i=1}^{N}\left[z_{i} b_{i}-\left|d_{i}\right|^{2} z_{i}\right]\right. \\
-\sum_{i=1}^{N} y_{i} \log \left(z_{i}\right)-\lambda \operatorname{Re}\left(\mathbf{z}^{\mathrm{H}} \mathbf{D}^{\mathrm{H}} \mathbf{A}\left(\mathbf{A}^{\mathrm{H}} \mathbf{A}\right)^{-1} \mathbf{T}^{\mathrm{H}} \mathbf{w}\right) \\
\left.+\frac{\lambda^{2}}{4} \mathbf{w}^{\mathrm{H}}(\mathbf{X}-e \mathbf{I}) \mathbf{w}+\frac{\lambda^{2}}{4} e\|\mathbf{w}\|_{2}^{2}\right) .
\end{array}
$$

The terms $\mathbf{z}^{\mathrm{H}} \mathbf{D}^{\mathrm{H}}(\mathbf{P}-\mathbf{I}) \mathbf{D} \mathbf{z}$ and $\frac{\lambda^{2}}{4} \mathbf{w}^{\mathrm{H}}(\mathbf{X}-e \mathbf{I}) \mathbf{w}$ in (57) are concave functions in $\mathbf{z}$ and $\mathbf{w}$, respectively and therefore 
are linearized to give the following surrogate minimization problem (the surrogate objective would be tighter at $\left(\mathbf{z}_{k}, \mathbf{w}_{k}\right)$ ):

$$
\begin{aligned}
\max _{\substack{\mathbf{z} \geq 0,\left|w_{l}\right| \leq 1}} & \left(2 \operatorname{Re}\left(\mathbf{z}_{k}^{\mathrm{H}} \mathbf{D}^{\mathrm{H}}(\mathbf{P}-\mathbf{I}) \mathbf{D z}\right)+\mathbf{z}^{\mathrm{H}} \mathbf{D}^{\mathrm{H}} \mathbf{D} \mathbf{z}-\sum_{i=1}^{N} z_{i}\left|d_{i}\right|^{2}\right. \\
& -\sum_{i=1}^{N} y_{i} \log \left(z_{i}\right)-\lambda \operatorname{Re}\left(\mathbf{z}^{\mathrm{H}} \mathbf{D}^{\mathrm{H}} \mathbf{A}\left(\mathbf{A}^{\mathrm{H}} \mathbf{A}\right)^{-1} \mathbf{T}^{\mathrm{H}} \mathbf{w}\right) \\
& \left.+\frac{\lambda^{2}}{2} \operatorname{Re}\left(\mathbf{w}_{k}^{\mathrm{H}}(\mathbf{X}-e \boldsymbol{I}) \mathbf{w}\right)+\frac{\lambda^{2}}{4} e\|\mathbf{w}\|_{2}^{2}+\sum_{i=1}^{N} z_{i} b_{i}\right) .
\end{aligned}
$$

Problem (58) can be solved by alternatingly minimizing it with respect to $\mathbf{z}$ and $\mathbf{w}$ [45]. Keeping $\mathbf{w}_{k}$ fixed, we first solve for solve for $\mathbf{z}_{k+1}$ as follows:

$$
\begin{array}{r}
\min _{\mathbf{z} \geq 0}\left(2 \operatorname{Re}\left(\mathbf{z}_{k}^{\mathrm{H}} \mathbf{D}^{\mathrm{H}}(\mathbf{P}-\mathbf{I}) \mathbf{D} \mathbf{z}\right)+\sum_{i=1}^{N}\left[z_{i} b_{i}-z_{i}\left|d_{i}\right|^{2}-y_{i} \log \left(z_{i}\right)\right]\right. \\
\left.+\mathbf{z}^{\mathrm{H}} \mathbf{D}^{\mathrm{H}} \mathbf{D} \mathbf{z}-\lambda \operatorname{Re}\left(\mathbf{z}^{\mathrm{H}} \mathbf{D}^{\mathrm{H}} \mathbf{A}\left(\mathbf{A}^{\mathrm{H}} \mathbf{A}\right)^{-1} \mathbf{T}^{\mathrm{H}} \mathbf{w}_{k}\right)\right) .
\end{array}
$$

With $\mathbf{c} \triangleq 2 \operatorname{Re}\left(\mathbf{D}^{\mathrm{H}}(\mathbf{P}-\mathbf{I}) \mathbf{D} \mathbf{z}_{k}\right)$ and $\mathbf{g} \triangleq$ $\lambda \operatorname{Re}\left(\mathbf{w}_{k}^{\mathrm{H}} \mathbf{T}\left(\mathbf{A}^{\mathrm{H}} \mathbf{A}\right)^{-1} \mathbf{A}^{\mathrm{H}} \mathbf{D}\right)$, the minimization problem over $\mathbf{z}$ becomes separable in $z_{i}$ as shown below:

$$
\min _{z_{i} \geq 0} \sum_{i=1}^{N}\left[c_{i} z_{i}+h_{i} z_{i}^{2}-g_{i} z_{i}+z_{i} b_{i}-y_{i} \log \left(z_{i}\right)-h_{i} z_{i}\right] \text {. }
$$

Thus a generic problem in one variable (without the index $i$ ) can be written as

$$
\min _{z \geq 0} c z+h z^{2}-g z+z b-y \log (z)-h z .
$$

Writing KKT condition and solving it we get the optimal solution as:

$$
z_{k+1}= \begin{cases}\frac{-(b+c-g-h)+\sqrt{(b+c-g-h)^{2}+8 h y}}{4 h}, & \text { if } h \neq 0 \\ \frac{y}{b+c-g}, & \text { if } h=0\end{cases}
$$

Now for a fixed $\mathbf{z}_{k+1}$, we solve for $\mathbf{w}_{k+1}$ as follows:

$$
\begin{array}{r}
\min _{\left|w_{l}\right| \leq 1}\left(\frac{\lambda^{2}}{2} \operatorname{Re}\left(\mathbf{w}_{k}^{\mathrm{H}}(\mathbf{X}-e \mathbf{I}) \mathbf{w}\right)+\frac{\lambda^{2}}{4} e\|\mathbf{w}\|_{2}^{2}\right. \\
\left.-\lambda \operatorname{Re}\left(\mathbf{z}_{k+1}^{\mathrm{H}} \mathbf{D}^{\mathrm{H}} \mathbf{A}\left(\mathbf{A}^{\mathrm{H}} \mathbf{A}\right)^{-1} \mathbf{T}^{\mathrm{H}} \mathbf{w}\right)\right) .
\end{array}
$$

By scaling out the factor $\frac{\lambda^{2}}{4} e$, we get:

$$
\begin{array}{r}
\min _{\left|w_{l}\right| \leq 1}\|\mathbf{w}\|_{2}^{2}-2 \operatorname{Re}\left(\frac{2}{\lambda e} \mathbf{z}_{k+1}^{\mathrm{H}} \mathbf{D}^{\mathrm{H}} \mathbf{A}\left(\mathbf{A}^{\mathrm{H}} \mathbf{A}\right)^{-1} \mathbf{T}^{\mathrm{H}} \mathbf{w}\right. \\
\left.-\frac{1}{e} \mathbf{w}_{k}^{\mathrm{H}}(\mathbf{X}-e \mathbf{I}) \mathbf{w}\right) .
\end{array}
$$

With $\mathbf{u} \triangleq \frac{2}{\lambda e} \mathbf{T}\left(\mathbf{A}^{\mathrm{H}} \mathbf{A}\right)^{-1} \mathbf{A}^{\mathrm{H}} \mathbf{D} \mathbf{z}_{k+1}-\frac{1}{e}(\mathbf{X}-e \mathbf{I})^{\mathrm{H}} \mathbf{w}_{k}$, the above problem also becomes separable in $w_{l}$

$$
\min _{\left|w_{l}\right| \leq 1} \sum_{l=1}^{N}\left|w_{l}\right|^{2}-2 \operatorname{Re} \sum_{l=1}^{N}\left(u_{l}^{*} w_{l}\right) .
$$

Therefore, a generic problem can be written as

$$
\min _{|w| \leq 1}|w|^{2}-2 \operatorname{Re}\left(u^{*} w\right)
$$

The problem (66) has a closed form solution and the optimal value of $w$ is given as

$$
w_{k+1}= \begin{cases}u & \text { if }|u| \leq 1 \\ u /|u| & \text { if }|u|>1\end{cases}
$$

The pseudo code for the MM algorithm for the regularized problem is given in Algorithm Table 2.

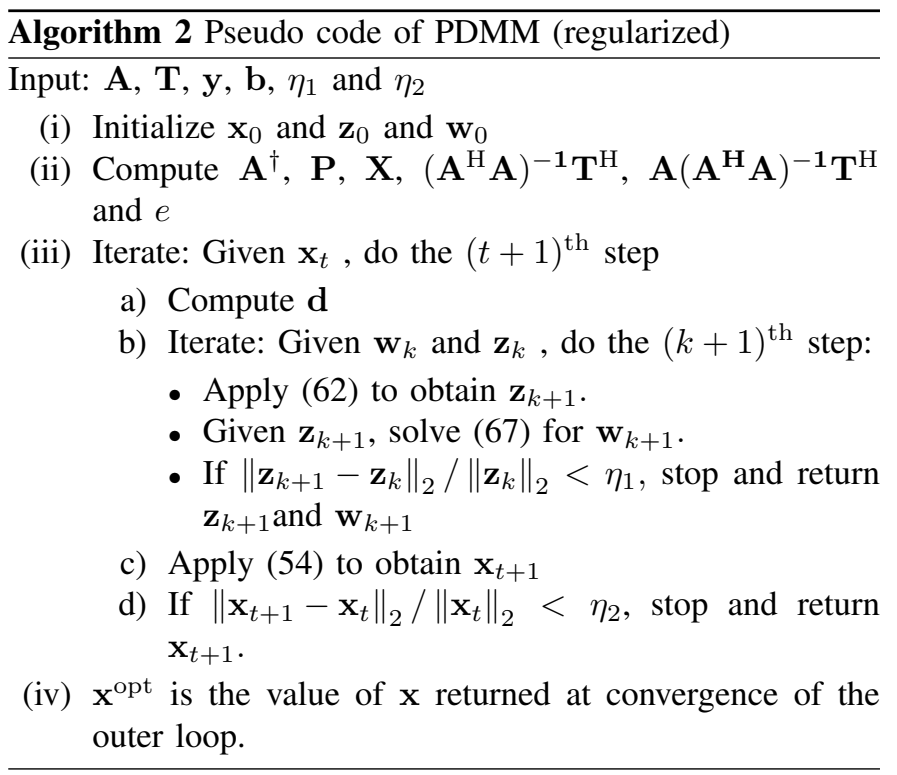

\section{Computational Complexity of PDMM}

For the unregularized problem, the main computational overheads of PDMM are in the calculation of pseudo inverse $\mathbf{A}^{\dagger}$ (complexity $O\left(K^{2} N\right)$ ) and the projection matrix $\mathbf{P}$ (complexity of $O\left(N^{2} K\right)$ ), assuming $N>K$, both of which are calculated outside the loops. Thus the per iteration computational complexity of PDMM is dominated only by some matrixvector multiplications (with a worst case complexity $O(N K)$ ) and Hadamard products of vectors (with a complexity $O(N)$ ) in the calculation of vectors $\mathbf{c}, \mathbf{d}, \mathbf{z}$ and $\mathbf{x}$.

For the regularized problem, the pseudo inverse matrix $\mathbf{A}^{\dagger}$, the projection matrix $\mathbf{P}$ as well as the matrices $\left(\mathbf{A}^{\mathrm{H}} \mathbf{A}\right)^{-1} \mathbf{T}^{\mathrm{H}}$ (complexity of $\left.O\left(K^{2} N\right)\right), \mathbf{A}\left(\mathbf{A}^{\mathrm{H}} \mathbf{A}\right)^{-1} \mathbf{T}^{\mathrm{H}}$ (complexity of $O\left(K^{2} N\right)$ ), $\mathbf{X}$ (complexity of $O\left(K^{2} N\right)$ ) and maximum eigenvalue $e$ (complexity of $O\left(K^{3}\right)$ ) are calculated and stored outside the loops. For large dimensional problems, instead of the eigenvalue $e$, the trace of the matrix $\mathbf{X}$ can be used which would result in a looser upperbound and a slower converging algorithm; however, would reduce the overall computational complexity. The per-iteration computational complexity in the regularized PDMM algorithm is only dominated by matrixvector multiplication (with a worst case complexity $O(N K)$ ) and Hadamard products of vectors (with a complexity $O(N)$ ) in the calculation of vectors $\mathbf{c}, \mathbf{d}, \mathbf{g}, \mathbf{z}, \mathbf{w}$ and $\mathbf{x}$. The complexity in the regularized case is greater than the unregularized case because of the calculation of more number of parameters.

In the case of a DFT matrix setting, none of the quantities are pre-calculated and stored, but are invoked inside the loops along with other calculations using Fast Fourier Transform (FFT) and Inverse Fast Fourier Transform (IFFT) which further reduces the computational complexity of the algorithm.

\section{E. Convergence Analysis of PDMM}

In this sub-section, we will prove that the iterative steps of PDMM always converges to a stationary point of the 
Poisson likelihood problem. Since PDMM is a double loop MM algorithm, we will prove the convergence of both the MM updates separately. Moreover, the convergence of the MM update over primal variable $\mathbf{x}$ depends on the convergence of the MM update over dual variable $\mathbf{z}$ (minimization problem in (38)). Therefore, we first prove the convergence of the MM update over dual variable $\mathbf{z}$.

Let us rewrite the objective function of the dual problem in (38) as:

$$
\begin{array}{r}
p(\mathbf{z})=\mathbf{z}^{\mathrm{H}} \mathbf{D}^{\mathrm{H}} \mathbf{A}\left(\mathbf{A}^{\mathrm{H}} \mathbf{A}\right)^{-1} \mathbf{A}^{\mathrm{H}} \mathbf{D} \mathbf{z}+\sum_{i=1}^{N} z_{i} b_{i} \\
-\sum_{i=1}^{N} y_{i} \log \left(z_{i}\right)-\sum_{i=1}^{N} z_{i}\left|d_{i}\right|^{2}
\end{array}
$$

As explained in subsection III-A, the sequence of points $\left\{\mathbf{z}_{k}\right\}$ generated by MM update results in a monotonically decreasing objective function $(p(\mathbf{z}))$. It can be seen that $p(\mathbf{z})$ is convex and a continuous function, and also bounded from below since for finite values of $y_{i}, b_{i}$ and $d_{i}$, the objective function $p\left(z_{i}\right) \rightarrow \infty$ at the extremum points of the function i.e. $z_{i} \rightarrow \infty$ and/or $z_{i} \rightarrow 0$. Therefore, the sequence generated by MM algorithm $\left\{p\left(\mathbf{z}_{k}\right)\right\}$ converges to some finite value. From (19) we have

$$
p\left(\mathbf{z}_{0}\right) \geq p\left(\mathbf{z}_{1}\right) \geq p\left(\mathbf{z}_{2}\right) \ldots
$$

We assume that there exists a convergent subsequence $\left\{\mathbf{z}_{k_{j}}\right\}$ which converges to the limit point $\mathbf{z}_{\text {limit }}$. We next prove that $\mathbf{z}_{\text {limit }}$ is a stationary point. Since the dual problem is a constrained problem, for a point $\mathbf{z}_{\text {limit }}$ to be stationary, it needs to satisfy:

$$
\operatorname{Re}\left(\nabla p\left(\mathbf{z}_{\text {limit }} ; \mathbf{d}\right)^{\mathrm{H}} \mathbf{t}\right) \geq 0 \forall \mathbf{t} \in T_{\mathbb{R}_{+}^{n}}\left(\mathbf{z}_{\text {limit }}\right)
$$

where $T_{\mathbb{R}_{+}^{n}}\left(\mathbf{z}_{\text {limit }}\right)$ denotes the tangent cone of $\mathbb{R}_{+}^{n}$ (constraint set of $\mathbf{z})$ at $\mathbf{z}_{\text {limit }}$ and $\nabla p(\mathbf{z} ; \mathbf{d})$ is the directional derivative of the function $p(\mathbf{z})$ which is defined as:

$$
\nabla p(\mathbf{z} ; \mathbf{d})=\lim _{\alpha \rightarrow 0} \inf \frac{p(\mathbf{z}+\alpha \mathbf{d})-p(\alpha)}{\alpha}
$$

Let $g_{p}($.$) denote the surrogate function for the function p(\mathbf{z})$ (which is the objective of (38)). Using (19), we get the following inequality:

$$
\begin{array}{r}
g_{p}\left(\mathbf{z}_{k_{j+1}} \mid \mathbf{z}_{k_{j+1}}\right)=p\left(\mathbf{z}_{k_{j+1}}\right) \leq p\left(\mathbf{z}_{k_{j}+1}\right) \\
\leq g_{p}\left(\mathbf{z}_{k_{j}+1} \mid \mathbf{z}_{k_{j}}\right) \leq g_{p}\left(\mathbf{z} \mid \mathbf{z}_{k_{j}}\right)
\end{array}
$$

For a limit point, $j \rightarrow \infty$, and we get:

$$
g_{p}\left(\mathbf{z}_{\text {limit }} \mid \mathbf{z}_{\text {limit }}\right) \leq g_{p}\left(\mathbf{z} \mid \mathbf{z}_{\text {limit }}\right)
$$

which implies

$$
\operatorname{Re}\left(\nabla g_{p}\left(\mathbf{z}_{\text {limit }}\right)^{\mathrm{H}} \mathbf{t}\right) \geq 0 \forall \mathbf{t} \in T_{\mathbb{R}_{+}^{n}}\left(\mathbf{z}_{\text {limit }}\right)
$$

Since the first order behavior of $p(\mathbf{z})$ is same as that of $g_{p}(\mathbf{z})$, we get

$$
\operatorname{Re}\left(\nabla p\left(\mathbf{z}_{\text {limit }}\right)^{\mathrm{H}} \mathbf{t}\right) \geq 0 \forall \mathbf{t} \in T_{\mathbb{R}_{+}^{n}}\left(\mathbf{z}_{\text {limit }}\right)
$$

thereby proving that $\mathbf{z}_{\text {limit }}$ is a stationary point of $p(\mathbf{z})$. Since $p(\mathbf{z})$ is a strongly convex function, $\mathbf{z}_{\text {limit }}$ would also be a global minimizer of $p(\mathbf{z})$.
The convergence of the MM algorithm over primal variable $\mathbf{x}$ can be proved in a similar way. Let us recollect the objective function of the primal problem as:

$$
f(\mathbf{x})=\sum_{i=1}^{N}\left[\left|\mathbf{a}_{i}^{\mathrm{H}} \mathbf{x}\right|^{2}+b_{i}-y_{i} \log \left(\left|\mathbf{a}_{i}^{\mathrm{H}} \mathbf{x}\right|^{2}+b_{i}\right)\right]
$$

The sequence of points $\left\{\mathbf{x}_{t}\right\}$ generated by MM algorithm monotonically decreases the objective function $f(\mathbf{x})$ monotonically. Also, the objective function $f(\mathbf{x})$ is continuous and bounded from below since for a finite value of $y_{i}$, the objective function $f(\mathbf{x}) \rightarrow \infty$ at the extremum points of the function i.e. $x_{i} \rightarrow \infty$ and/or $x_{i} \rightarrow-\infty$, for any $i$. Therefore, the sequence generated by MM algorithm $\left\{f\left(\mathbf{x}_{t}\right)\right\}$ converges to some finite value at the limit point $\mathbf{x}_{\text {limit }}$. We next prove that $\mathbf{x}_{\text {limit }}$ is a stationary point. Since the primal problem is an unconstrained problem, for a point $\mathbf{x}$ to be stationary, $\nabla f(\mathbf{x} ; \mathbf{d})=0$. Similar to the argument used in the proof over dual variable $\mathbf{z}$, we get

$$
g_{f}\left(\mathbf{x}_{\text {limit }} \mid \mathbf{x}_{\text {limit }}\right) \leq g_{f}\left(\mathbf{x} \mid \mathbf{x}_{\text {limit }}\right)
$$

where $g_{f}($.$) denotes the surrogate function as in (28). The$ above result implies $\nabla g_{f}\left(\mathbf{x}_{\text {limit }}\right)=0$. Since the first order behavior of $f(\mathbf{x})$ is same as that of $g_{f}(\mathbf{x})$, we get $\nabla f\left(\mathbf{x}_{\text {limit }}\right)=0$, thereby proving that $\mathbf{x}_{\text {limit }}$ is a stationary point of (7). Thus, by proving the convergence of the MM updates over both primal and dual variables, we establish the proof of convergence of the proposed algorithm.

\section{NUMERICAL SIMULATIONS}

This section discusses the simulation details of the proposed algorithm under two different experimental settings. In the first setting, the matrix $\mathbf{A}$ is taken to be a complex random matrix with its elements having independent random real and imaginary parts following Uniform distribution in the interval $(0,1)$. In the second setting, the matrix $\mathbf{A}$ is modelled using the Discrete Fourier Transform (DFT) matrix. In case of random matrix setting, a random signal $\mathbf{x}_{\text {true }} \in \mathbb{C}^{\mathrm{K}}$, normalized as $\mathbf{x}_{\text {true }} /\left\|\mathbf{x}_{\text {true }}\right\|_{2}$, is taken as the original signal. Whereas, for the DFT matrix setting, an image of size $K \times K$ is the original signal. The background signal $b$ in both the settings is a constant vector with values equal to 0.1 for the case $b_{i}>0$. All the measurements are considered to be corrupted with noise (n) following Poisson distribution. The measurement vector $\mathbf{y}$ is modelled as $\mathbf{y}=\left|\mathbf{A} \mathbf{x}_{\mathrm{o}}\right|^{2}+\mathbf{b}+\mathbf{n}$. The performance of the proposed algorithm (PDMM) is compared with the competing algorithms such as WF, ADMM, and MM proposed in [37]. Since the authors of [37] have already compared their proposed algorithm with the GS algorithm and WF (Gaussian) algorithm and have established that the Poisson phase-retrieval algorithms perform better than the algorithms for Gaussian model in case of Poisson data model, we do not include the comparison of PDMM with the Gaussian phaseretrieval algorithms in this paper.

The experiments are conducted using MATLAB (R2018a) on a personal computer with $1.7 \mathrm{GHz}$ Intel(R) Core(TM) i54210U CPU and 16.00 GB RAM. 


\section{A. Initialization and convergence threshold}

As suggested by the authors in [4], the leading eigenvector of the matrix $\mathbf{A}^{\mathrm{H}} \operatorname{diag}(\mathbf{y}-\mathbf{b}) \mathbf{A}$ is taken as an initial estimate $\tilde{\mathbf{x}}_{0}$. To tackle signals of arbitrary scale, the leading eigenvector obtained is scaled by a constant given by:

$$
\begin{aligned}
\hat{\alpha} & =\arg \min _{\alpha \in \mathbb{R}}\left\|\mathbf{y}-\mathbf{b}-\left|\alpha \mathbf{A} \tilde{\mathbf{x}}_{0}\right|^{2}\right\|_{2}^{2} \\
& =\frac{\sqrt{\left((\mathbf{y}-\mathbf{b})^{\mathrm{T}}\left|\mathbf{A} \tilde{\mathbf{x}}_{0}\right|^{2}\right)}}{\left\|\mathbf{A} \tilde{\mathbf{x}}_{0}\right\|_{4}^{2}} .
\end{aligned}
$$

Therefore, the initial estimate $\mathbf{x}_{0}$ is $\hat{\alpha} \tilde{\mathbf{x}}_{0}$. The vector $\mathbf{z}$ is initialized as $z_{i}=\frac{y_{i}}{\left|\mathbf{a}_{i}^{\mathrm{H}} \mathbf{x}_{0}\right|^{2}+b_{i}}$ and vector $\mathbf{w}$ is initialized as a random unit vector once outside the iteration loops.

The threshold $\eta_{1}$ for terminating the outer loop $\left(\frac{\left\|\mathbf{x}_{t+1}-\mathbf{x}_{t}\right\|_{2} \mid}{\left\|\mathbf{x}_{t}\right\|_{2}}<\eta_{1}\right)$ is fixed at $10^{-6}$ for every iteration. The threshold $\eta_{2}$ for terminating the the inner loop $\left(\frac{\left\|\mathbf{z}_{k+1}-\mathbf{z}_{k}\right\|_{2}}{\left\|\mathbf{z}_{k}\right\|_{2}}<\eta_{2}\right)$ can either be fixed and initialized outside the inner loop or can be adaptively changed after each iteration by checking for a decrease in the original objective function. (Please see the discussion in Remark 4).

\section{B. Ambiguities and Performance Evaluation}

Due to loss of global phase information, the phase-retrieval algorithms can recover the original signal only within a constant phase shift. Therefore in the case of random matrix setting, the following Normalized Root Mean Square Error (NRMSE) is used to evaluate the performance of proposed algorithm taking into consideration the global phase shift.

$$
\text { NRMSE }=\frac{\left\|\mathbf{x}^{\text {opt }}-\mathbf{x}_{\text {true }} \mathrm{e}^{\mathrm{i} \phi}\right\|_{2}}{\left\|\mathbf{x}_{\text {true }}\right\|_{2}}, \mathrm{e}^{\mathrm{i} \phi}=\operatorname{sign}\left(\mathbf{x}_{\text {true }}^{\prime} \mathbf{x}^{\text {opt }}\right),
$$

where $\mathrm{x}^{\mathrm{opt}}$ denotes the recovered signal and $\mathrm{x}_{\text {true }}$ denotes the original signal.

In case of DFT Matrix setting, there are more number of ambiguities like global constant phase shift, circular shift, conjugate inversion and their combinations that conserve the Fourier magnitude and contribute to trivial ambiguities. Also, two signals with the same autocorrelation function have the same Fourier magnitude. This results in the recovery of original signal only up to the same autocorrelation function without any additional information. One method to deal with these ambiguities is to use the mean squared error between the auto-correlation function of the original and recovered signal for performance evaluation instead of using NRMSE. Another method is to introduce redundancy in the measurement vector $\mathbf{y}$ using masked DFT. Here, instead of the measurements being made as:

$$
y_{n}=\left|\sum_{k=0}^{K-1} x_{k} \mathrm{e}^{-i 2 \pi n k / N}\right|^{2}+b_{n},
$$

where $N=2 K-1, M$ redundant masks $\mathbf{D}^{m}(m=1, \ldots, M)$ are introduced and the measurement model becomes:

$$
y_{n}^{m}=\left|\sum_{k=0}^{K-1} x_{k} D_{k}^{m} \mathrm{e}^{-i 2 \pi n k / N}\right|^{2}+b_{n}^{m} .
$$

Similar to [37], a total of $M=21$ masks (where the first mask is a full sampling mask and the rest have a sampling rate of 0.5 with random sampling patterns) are used in the experiments to define the measurement matrix $\mathbf{A} \in \mathbb{C}^{M N \times K}$.

\section{Experimental Settings}

This sub-section explains the details of different experiments performed under both random matrix Setting and DFT matrix setting.

1) Random Matrix Setting: Under random matrix setting, three different experiments are performed. In the first experiment, the length of $\mathbf{x}_{\text {true }}$ is fixed at $K=100$ and the average running time and NRMSE of the algorithms are plotted against the number of measurements $N$, where $N$ is varied between 1000 and 8000 at an interval of 1000 . In the second experiment, the number of measurements is fixed at $N=4000$ and the average running time and NRMSE of the algorithms are plotted against the length of $\mathbf{x}_{\text {true }}(K)$, where $K$ is varied between 100 and 500 at an interval of 100. In the third experiment, with the number of measurements fixed at $N=4000$ and the length of $\mathbf{x}_{\text {true }}$ fixed at $K=300$, the NRMSE is plotted against time. The plots of NRMSE against $N$ and $K$ gives the analysis of the accuracy of the proposed algorithm, whereas the plots of average running time against $N$ and $K$ gives an analysis of the convergence of the proposed algorithm. The NRMSE vs time plot gives an analysis of both accuracy and convergence. The experiments are repeated for 50 Monte-Carlo simulations to calculate the average computational time and NRMSE values. All the experiments are performed for both when the background signal $b_{i}=0$ and $b_{i}>0$.

2) DFT Matrix Setting: In many phase retrieval problems such as those arising in optical imaging, the measurements are the magnitudes of the Fourier transform. The measurement matrix $\mathbf{A}$ in such cases is modelled as a DFT matrix. Therefore, in the second experimental setting, we test the proposed algorithm on an image of size $K \times K$, where the measurement matrix $\mathbf{A}$ is as described in the previous subsection with $M=21$ masked DFT matrices. The matrix $\mathbf{A}$ is normalized such that the average of $\left|\mathbf{a}_{i}^{\mathrm{H}} \mathbf{x}\right|^{2}$ is 1 for $i=1, \ldots, N$. The Cameraman image of size $128 \times 128$ pixels is used as the original image. To demonstrate that PDMM can be adapted to solve $\ell_{1}$ regularized problems, where the matrix $\mathbf{T}$ may not be proximal friendly, the experiments under DFT matrix setting are performed for TV regularized Poisson phase retrieval problem. The reconstructed image along with the corresponding NRMSE is compared with the original image. Furthermore, NRMSE is plotted against time for the analysis of the convergence of the PDMM. The results of PDMM is also compared with ADMM-TV and MM-TV algorithms as proposed in [37] which use alternating minimization and Conjugate Gradient method in their iterations to solve for the optimal value of $\mathbf{x}$. The WF algorithm proposed in [37] is not considered for comparison in the case of DFT matrix setting because gradient based methods like WF are not suitable for non-smooth $\ell_{1}$ regularizers. Similar to [37], the value of regularization parameter $(\lambda)$ is chosen to be 8 . 


\section{SIMULATION RESULTS}

This section gives the simulation results of the experiments for both random matrix setting and DFT matrix setting and compares the result with the competing algorithms such as the WF, MM and ADMM algorithms for Poisson distribution as proposed in [37].

1) Random Matrix Setting:

(i) Average time vs Number of measurements $(N)$

Fig. 2 shows the comparison of average running time against the number of measurements $(N)$ for all algorithms. Sub-Figures (a) and (b) show results for cases when background signal $b_{i}=0.1$ and $b_{i}=0$ respectively. It is observed that PDMM is faster than the competing algorithms.

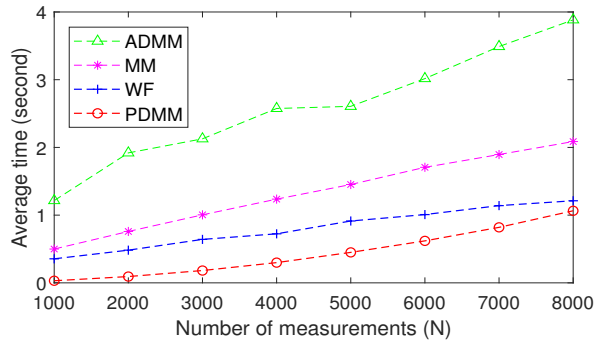

(a)

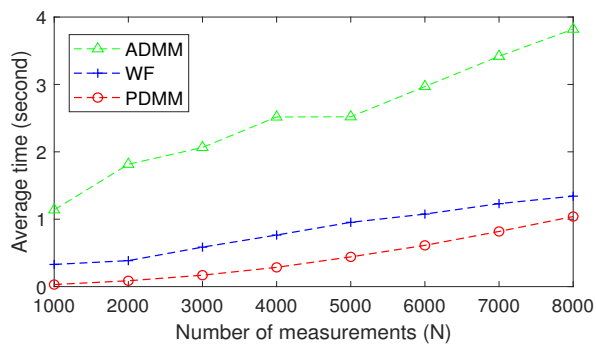

(b)

Fig. 2. Average computation time (in seconds) vs Number of measurements $N$, when $\eta=10^{-6}, \mathbf{x} \in \mathbb{C}^{100}$ and $\mathbf{A}$ is a random matrix. Sub-Figures (a) and (b) corresponds to cases when $b_{i}=0.1$ and $b_{i}=0$ respectively.

(ii) NRMSE vs Number of measurements $(N)$

Fig. 3 gives the comparison of the NRMSE against the number of measurements $(N)$ for all algorithms. The plot for PDMM overlaps almost completely with the WF, ADMM and MM algorithms. Thus, in terms of the accuracy of the recovered signal, the performance of PDMM is at par with the previously proposed algorithms.

(iii) Average time vs Length of the original signal $(K)$

Fig. 4 compares the plots of average running time against the length of the original signal $(K)$ for all algorithms. For a fixed number of measurements, PDMM is the fastest for different lengths of $\mathbf{x}_{\text {true }}$. The computational complexity of the MM algorithm increases with the increase in the length of $x_{\text {true }}$ perhaps due to the calculation of inverse of a $K \times K$ matrix performed at every iteration of the algorithm. This can be reduced by

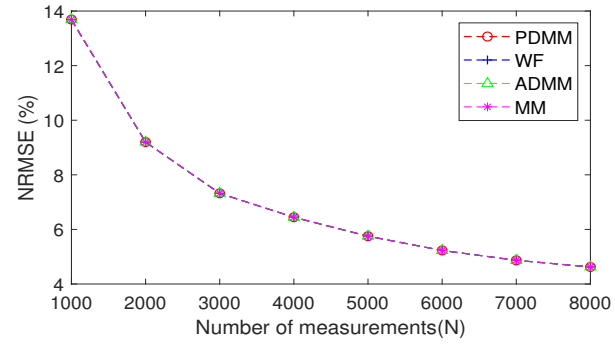

(a)

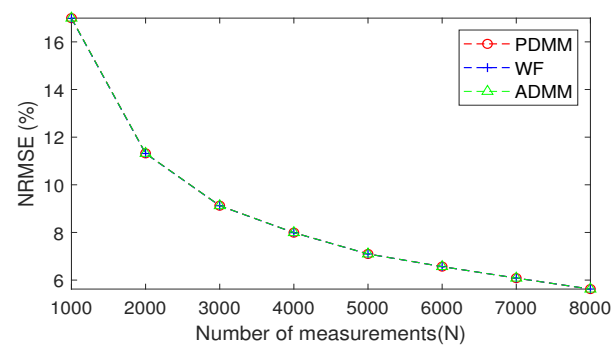

(b)

Fig. 3. NRMSE vs Number of measurements $(N)$ for $\eta=10^{-6}, \mathbf{x} \in \mathbb{C}^{100}$ and $\mathbf{A}$ is a random matrix. Sub-Figure (a) and (b) corresponds to the cases when $b_{i}=0.1$ and $b_{i}=0$ respectively.

using CG for updating $\mathbf{x}$. The WF algorithm converges slowly for larger values of $K$.

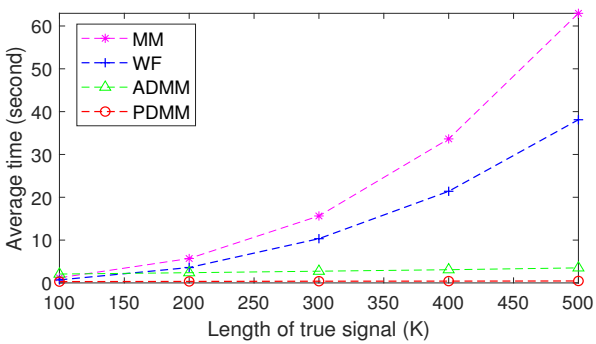

(a)

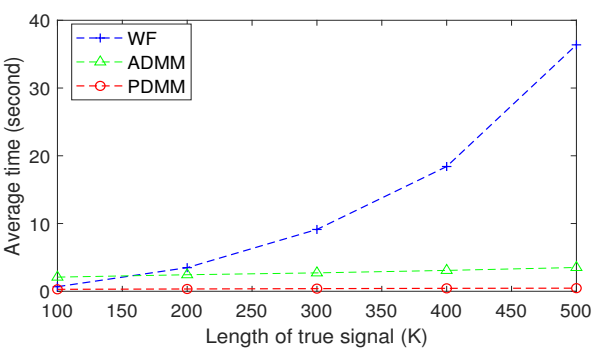

(b)

Fig. 4. Average computation time (in seconds) vs Length of original signal $K$, when $\eta=10^{-6}, N=4000$ and $\mathbf{A}$ is a random matrix. Sub-Figures (a) and (b) corresponds to cases when $b_{i}=0.1$ and $b_{i}=0$ respectively.

(iv) NRMSE vs Length of original signal $(K)$

Fig. 5 gives the comparison of the plots of NRMSE against the length of $\mathbf{x}_{\text {true }}(K)$ for each algorithm. As expected, the NRMSE increases with $K$ because of increase in the number of parameters estimated. The 
plot for PDMM overlaps almost completely with WF, ADMM and MM algorithm indicating its performance to be at par with the previously proposed algorithms in terms of successful recovery of the original signal.

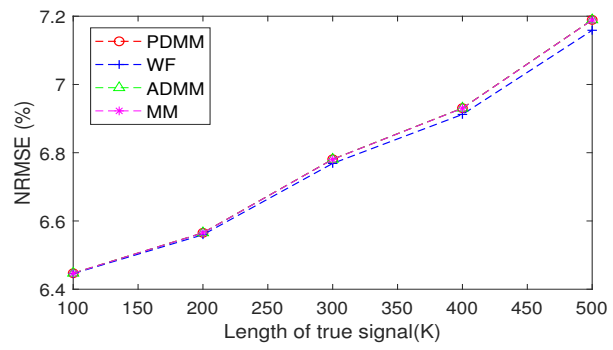

(a)

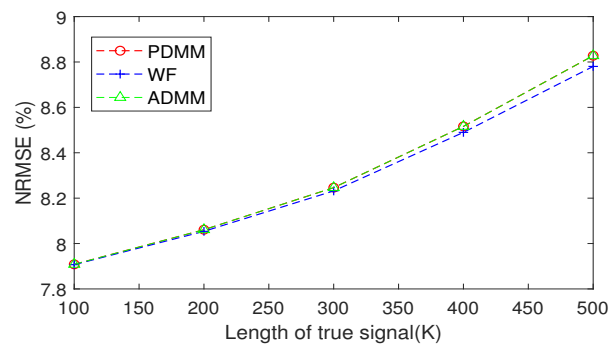

(b)

Fig. 5. NRMSE vs Length of original signal $(K)$ for $\eta=10^{-6}, N=4000$ and $\mathbf{A}$ being a random matrix. Sub-Figure (a) and (b) corresponds to the cases when $b_{i}=0.1$ and $b_{i}=0$ respectively.

(v) NRMSE vs Time

Fig. 6 gives the NRMSE vs time plots for all the algorithms. The number of measurements is fixed at $N=4000$ and the length of $\mathbf{x}_{\text {true }}$ is 300 . It is observed that PDMM converges faster than the other three algorithms.

2) DFT Matrix Setting: The experiments under DFT matrix setting were performed for the TV regularized Poisson likelihood problem. Fig. 7 compares the result of PDMM and other algorithms for Cameraman image of size $128 \times 128$ pixels. The figure shows the original image along with the recovered images using PDMM, ADMM and MM algorithm. The corresponding NRMSE is mentioned below each image. Fig. 8 gives NRMSE vs time plots for all the algorithms for the said image. The speed of the proposed algorithm is comparable to the MM algorithm and is much faster than the ADMM algorithm proposed in [37]. In terms of recovery of the original image, its performance is at par with the state-ofthe-art algorithms.

\section{CONCLUSION}

This paper introduces a novel method for Poisson phaseretrieval based on the MM framework. In this method, using Fenchel representation of the log term, an auxiliary dual variable is introduced and the problem is converted into a saddle-point minimax problem. Surrogate functions over both primal and dual variables are proposed, resulting in a double loop MM algorithm. The resulting primal-dual majorizationminimization (PDMM) algorithm is compared against the existing maximum likelihood (ML) estimation algorithms for solving the Poisson phase-retrieval problem. It is observed that the proposed algorithm (PDMM) is in general faster than the algorithms proposed in [37]. The performance of PDMM in terms of the accuracy of recovered signal/image is at par with previously proposed algorithms. Unlike the other algorithms, the proposed algorithm can be easily adapted to regularized problem, where the regularizer may not be smooth and proximal friendly. Furthermore, the previously proposed MM algorithm, where a quadratic majorizer is used, works only in cases where background signal $b_{i}>0$, whereas PDMM works even when $b_{i}=0$.

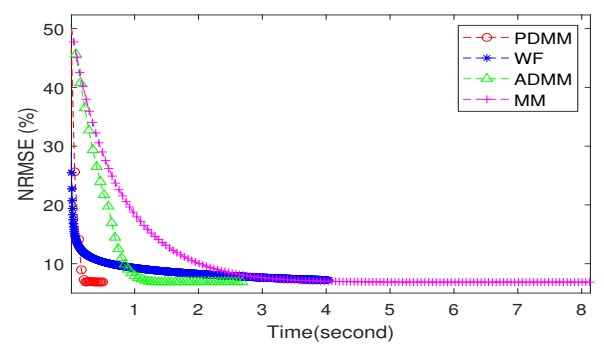

(a)

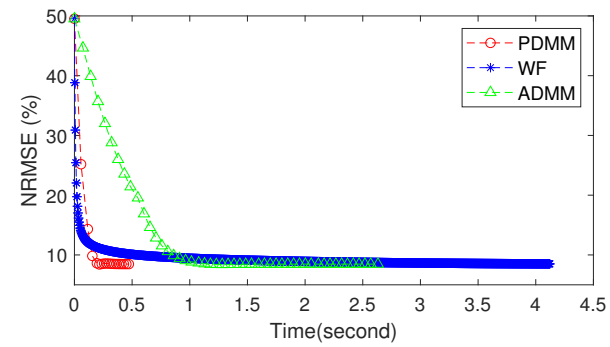

(b)

Fig. 6. NRMSE vs time when $N=4000, \mathbf{x} \in \mathbb{C}^{300}, \eta=10^{-6}$ and $\mathbf{A}$ is a random matrix. Sub-Figure (a) and (b) corresponds to the cases when $b_{i}=0.1$ and $b_{i}=0$ respectively.

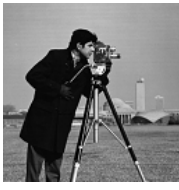

(a) True Image

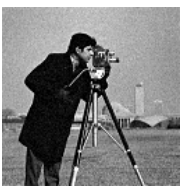

(b) PDMM $(6.3 \%)$

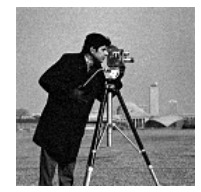

(c) $\operatorname{ADMM}(6.9 \%)$

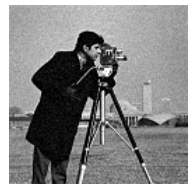

(d) $\mathrm{MM}(7.5 \%)$
Fig. 7. Reconstructed image and corresponding NRMSE compared to the true image (Cameraman of size $128 \times 128$ ), for a measurement system with $M=21$ masked DFT matrices and TV regularized phase-retrieval problem.

\section{ACKNOWLEDGEMENT}

We would like to thank Professor Jeffrey A. Fessler for his feedback on the draft. 


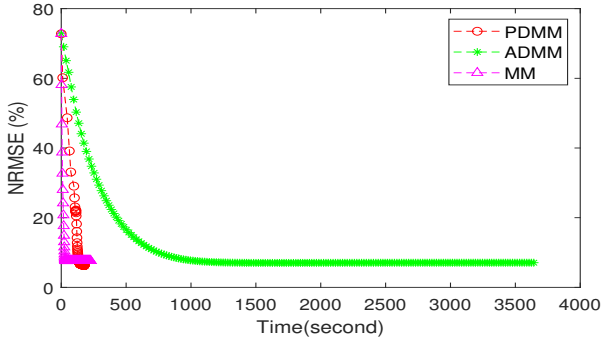

Fig. 8. NRMSE vs time plot for a $128 \times 128$ Cameraman image for a measurement system with $M=21$ masked DFT matrices and TV regularized phase-retrieval problem.

\section{REFERENCES}

[1] K. Jaganathan, Y. C. Eldar, and B. Hassibi, "Phase retrieval: an overview of recent developments," Optical Compressive Imaging, pp. 279-312, 2016.

[2] A. V. Oppenheim and J. S. Lim, "The importance of phase in signals," Proceedings of the IEEE, vol. 69, no. 51, pp. 529-541, 1981.

[3] A. Walther, "The question of phase retrieval in optics," Optica Acta: Int. J. Opt., vol. 10, no. 1, pp. 41-49, 1963. [Online]. Available: http://dx.doi.org/10.1080/713817747.

[4] J. Dainty and J. Fienup, "Phase retrieval and image reconstruction for astronomy", Imag. Recov. Theory Appl., vol. 13, pp. 231-275, Jan. 1987.

[5] M. Hayes, "The reconstruction of a multidimensional sequence from the phase or magnitude of its Fourier transform", IEEE Trans. Acoust., Speech, Signal Proc., vol. 30, pp. 140-154, 1982.

[6] N. Sturmel and L. Daudet, "Signal reconstruction from STFT magnitude: A state of the art," in Proc. Int. Conf. Digit. Audio Effects DAFx, vol. 2012, pp. 375-386, 2011.

[7] J. Le Roux and E. Vincent, "Consistent Wiener filtering for audio source separation," IEEE Signal Process. Lett., vol. 20, no. 3, pp. 217-220, Mar. 2013.

[8] T. Gerkmann, M. Krawczyk-Becker, and J. Le Roux, "Phase processing for single-channel speech enhancement: History and recent advances," IEEE Signal Process. Mag., vol. 32, no. 2, pp. 55-66, Mar. 2015.

[9] H. Hauptman, "The direct methods of X-ray crystallography," Science, vol. 233, no. 4760, pp. 178-183, 1986

[10] R.P. Millane, "Phase retrieval in crystallography and optics," J. Opt. Soc. Am. A., vol. 7, pp. 394-411, 1990

[11] R. W. Harrison, "Phase problem in crystallography," J. Opt. Soc. Amer. $A$, vol. 10, no. 5, pp. 1046-1055, May 1993. [Online]. Available: http://josaa.osa.org/abstract.cfm?URI=josaa-10-5-1046.

[12] M. Stek, "Inferring DNA structures from segmentation data," Artificial Intelligence, vol. 11, no. 1-2, pp. 85-114, 1978.

[13] D.L. Misell, "A method for the solution of the phase problem in electron microscopy," J. Phys. D: App. Phy., vol. 6, no. 1, pp. L6-L9, 1973.

[14] J. Miao, T. Ishikawa, B. Johnson, E. H. Anderson, B. Lai, and K. O. Hodgson, "High resolution 3d x-ray diffraction microscopy," Phys. Rev. Lett., vol. 89, p. 088303, Aug. 2002. [Online]. Available: http://link.aps.org/doi/10.1103/PhysRevLett.89.088303

[15] Y. J. Liu, B. Chen, E. R. Li, Y. J. Wang, A. Marcelli, S. W. Wilkins, H. Ming, Y. C. Tian, K. A. Nugent, P. P. Zhu, and Z. Y. Wu, "Phase retrieval in X-ray imaging based on using structured illumination," Physical Review A, vol. 78, no. 2, pp. 023817, 2008.

[16] E. J. Cand'es, T. Strohmer, and V. Voroninski, "Phaselift: Exact and stable signal recovery from magnitude measurements via convex programming," Commun. Pure Appl. Math., vol. 66, no. 8, pp. 1241-1274, 2013.

[17] A. S. Bandeira, J. Cahill, D. G. Mixon, and A. A. Nelson, "Saving phase: Injectivity and stability for phase retrieval," Appl. Comput. Harmon. Anal., vol. 37, no. 1, pp. 106-125, 2014. [Online]. Available: http://www.sciencedirect.com/science/article/pii/S1063520313000936.

[18] E. Candes, Y. Eldar, T. Strohmer, and V. Voroninski, "Phase retrieval via matrix completion," SIAM J. Imag. Sci., vol. 6, no. 1, pp. 199-225, 2013. [Online]. Available: http://dx.doi.org/10.1137/110848074.

[19] Y. Shechtman, Y. Eldar, O. Cohen, H. Chapman, J. Miao, and M. Segev, "Phase retrieval with application to optical imaging: A contemporary overview," IEEE Signal Process. Mag., vol. 32, no. 3, pp. 87-109, May 2015.
[20] E. Candes, X. Li, and M. Soltanolkotabi, "Phase Retrieval via Wirtinger Flow: Theory and Algorithms", IEEE Trans. Info. Theory, vol. 61, no. 4, pp. 1985-2007, Apr. 2015.

[21] T. Qiu, P. Babu, and D. P. Palomar, "PRIME: phase retrieval via majorization-minimization", IEEE Trans. Sig. Proc, vol. 64, no. 19, 5174-86, Oct. 2016.

[22] R. W. Gerchberg and W. O. Saxton, "A practical algorithm for the determination of phase from image and diffraction plane pictures," Optik, vol. 35 , p. 237, 1972.

[23] J. Liang, P. Stoica, Y. Jing, and Jian Li, "Phase retrieval via the alternating direction method of multipliers," IEEE Signal Processing Letters, vol. 25, no. 1, pp. 5-9, 2017.

[24] Y. Yang, M. Pesavento, Y. C. Eldar, and B. Ottersten, "Parallel coordinate descent algorithms for sparse phase retrieval." in ICASSP 20192019 IEEE International Conference on Acoustics, Speech and Signal Processing (ICASSP), pp. 7670-7674, IEEE, 2019.

[25] I. Waldspurger, A. d'Asspremont, and S. Mallat, "Phase recovery, Maxcut and complex semidefinite programming," Math. Programm., vol. 149, no. 1-2, pp. 47-81, 2015. [Online]. Available: http://dx.doi. org/10.1007/s10107-013-0738-9.

[26] P. Thibault and M. Guizar-Sicairos, "Maximum-likelihood refinement for coherent diffractive imaging," New J. of Phys., vol. 14, no. 6, pp. 063004, June 2012.

[27] J.M. Rodenburg, "Ptychography and related diffractive imaging methods," Advances in Imaging and Electron Physics, vol. 150, pp. 87-184, 2008.

[28] M. Dierolf, A. Menzel, P. Thibault, P. Schneider, C. M. Kewish, R. Wepf, O. Bunk, and F. Pfeiffer, "Ptychographic X-ray computed tomography at the nanoscale," Nature, vol. 467, no. 7314, pp. 436-439, 2010.

[29] D. A. Barmherzig and J. Sun, "Low-photon holographic phase retrieval", In Computational Optical Sensing and Imaging, 2020, pp. JTu4A-6.

[30] A. Goy, K. Arthur, S. Li, and G. Barbastathis, "Low photon count phase retrieval using deep learning", Phys. Rev. Lett., vol. 121, no. 24, pp. 243902, Dec. 2018

[31] K. Choi and A. D. Lanterman, "Phase retrieval from noisy data based on minimization of penalized I-divergence," JOSA A, vol. 24, no. 1, pp. 34-49, Jan. 2007.

[32] L. Bian, J. Suo, J. Chung, X. Ou, C. Yang, F. Chen, and Q. Dai, "Fourier ptychographic reconstruction using Poisson maximum likelihood and truncated Wirtinger gradient " Nature Sci. Rep, vol. 6. no. 1, 2016

[33] E. J. Candes, Y. C. Eldar, T. Strohmer, and V. Voroninski, "Phase retrieval via matrix completion," SIAM review, vol. 57, no. 2, pp. 225$251,2015$.

[34] Y. Chen and E. J. Candes, "Solving random quadratic systems of equations is nearly as easy as solving linear systems," Comm. Pure Appl. Math., vol. 7, no. 5, pp.822-83, May 2017.

[35] H. Chang and S. Marchesini, "Denoising Poisson phaseless measurements via orthogonal dictionary learning," Optics Express, vol. 26, no. 16, pp. 19773-96, 2018.

[36] H. Chang, Y. Lou, Y. Duan, and S. Marchesini, "Total variation- based phase retrieval for poisson noise removal," SIAM journal on imaging sciences vol. 11 , no. 1 , pp. 24-55, 2018.

[37] Z. Li, K. Lange, and J. A. Fessler, "Algorithms for Poisson Phase Retrieval," arXiv preprint arXiv:2104.00861, 2021

[38] S. Boyd, S.P. Boyd, and L. Vandenberghe, "Convex Optimization", Cambridge University press, 2004.

[39] Y. Sun, P. Babu, and D. P. Palomar, "Majorization-Minimization Algorithms in Signal Processing, Communications, and Machine Learning," IEEE Transactions on Signal Processing, vol. 65, no. 3, pp. 794-816, 2017.

[40] M. Sion, "On general minimax theorems," Pacific journal of Mathematics, vol. 8, pp. 171-176, 1958.

[41] M Grant, and S. Boyd, CVX: Matlab software for disciplined convex programing, version 2.0 beta, September 2013. Available: http://cvxr.com/cvx

[42] K. Jaganathan, S. Oymak and B. Hassibi, "Sparse phase retrieval: Convex algorithms and limitations," in IEEE International Symposium on Information Theory Proceedings, pp. 1022-1026, 2013.

[43] T. Qiu and D. P. Palomar, "Undersampled Sparse Phase Retrieval via Majorization-Minimization," IEEE Transactions on Signal Processing, vol. 65, no. 22, pp. 5957-5969, 2017, doi: 10.1109/TSP.2017.2745459.

[44] E. J. R. Pauwels, A. Beck, Y. C. Eldar and S. Sabach, "On Fienup Methods for Sparse Phase Retrieval," IEEE Transactions on Signal Processing, vol. 66, no. 4, pp. 982-991, 2018, doi: 10.1109/TSP.2017.2780044.

[45] I. Csiszar, and G. Tusnady, "Information geometry and alternating minimization procedures," Statistics and Decisions, Supplement issue, vol. 1, pp. 205-237, 1984. 